\title{
Synchronised multi-scale image analysis of soil deformations
}

\section{AUTHOR:}

Teng, Y. ${ }^{1}$, Stanier, S. A. ${ }^{2}$ and Gourvenec, S. M. ${ }^{3}$

\section{POSITION AND AFFILIATION:}

${ }^{1}$ Research Student; BEng

${ }^{2}$ Research Fellow; MEng, PhD

${ }^{3}$ Professor, BEng, $\mathrm{PhD}$

All based at the Centre for Offshore Foundation Systems, University of Western Australia.

\section{CONTACT ADDRESS:}

Dr. Sam Stanier

Centre for Offshore Foundation Systems

M053 Fairway

Crawley

WA 6009

Australia

\section{NUMBER OF WORDS, FIGURES AND TABLES:}

Words: 6166

Figures: 15

\section{KEYWORDS:}

Image analysis, particle image velocimetry, digital image correlation, deformations 
Teng, Y., Stanier, S. A. and Gourvenec, S. M.

'Synchronised multi-scale image analysis of soil deformations'

International Journal of Physical Modelling in Geotechnics

Special edition on DIC. 17(1):53-71 http://dx.doi.org/10.1680/jphmg.15.00058

\title{
SYNCHRONISED MULTI-SCALE IMAGE ANALYSIS OF SOIL DEFORMATIONS
}

\author{
Teng, Y., Stanier, S. A. and Gourvenec, S. M.
}

\begin{abstract}
New apparatus and techniques for performing synchronised multi-scale Particle Image Velocimetry or Digital Image Correlation (PIV/DIC) soil deformation measurements have been developed. A central camera records a full FoV of the model capturing the 'macro' deformation mechanism and the boundaries of the model. Simultaneously, an adjacent slave camera records a subset of the full FoV capturing the 'micro' soil response in a region of special interest, such as under the corner of footing. The 'micro' FoV images have higher resolution in terms of particle/pixel size ratio $(d / p)$, resulting in the ability to measure localised deformations that are invisible to lower resolution images. Recommendations are made with respect to appropriate subset size and spacing for the high-resolution images. A photogrammetric correction process requiring a small number of static control points is proposed and the performance is validated against a conventional photogrammetric calibration utilising a large array of static control points. Lastly, results from a validation experiment are presented comparing PIV/DIC output from the 'macro' and 'micro' FoVs, illustrating: (i) that the photogrammetric correction method proposed is robust; and (ii) the improved spatial resolution of the strain measurements that can be obtained via the ‘micro' FoV camera.
\end{abstract}


Teng, Y., Stanier, S. A. and Gourvenec, S. M.

'Synchronised multi-scale image analysis of soil deformations'

International Journal of Physical Modelling in Geotechnics

Special edition on DIC. 17(1):53-71 http://dx.doi.org/10.1680/jphmg.15.00058

\section{INTRODUCTION}

This paper presents new synchronised multi-scale image analysis apparatus for observing soil deformations in a geotechnical centrifuge using Particle Image Velocimetry (PIV - a.k.a. Digital Image Correlation or DIC). The system is an extension of that described by Stanier and White (2013), which used a small form factor machine vision camera and bespoke software to provide in-flight live view of the images captured at rates of up to 15 frames per second. An additional camera was added to this system to enable synchronized images of two fields of view (FoV) to be captured simultaneously: one of the whole domain or the so-called 'macro' FoV and the other focusing on a smaller specific region of interest, the so-called 'micro' FoV. The 'micro' view images are of significantly higher resolution in terms of particle/pixel size ratio $(d / p)$ allowing localised deformations to be measured that are invisible to lower resolution images. Visualising soil behavior at the ‘micro’ scale, as well as at the 'macro’ scale, to identify macroscopic failure mechanisms, provides stronger experimental support for geotechnical analysis, and in particular the development of complex constitutive or discrete element models.

\section{BACKGROUND}

Particle image velocimetry (PIV) techniques (Adrian 1991, Westerweel et al. 1997) and digital image correlation (DIC) techniques (Sutton et al. 1983, Sutton et al. 2000) have been widely used for measuring deformation fields in physical modelling tests. Over the past fifteen years PIV/DIC techniques have been developed specifically for geotechnical applications (e.g. White et al. 2001, White et al. 2003, Rechenmacher and Finno 2004, Iskander 2010, Hall 2012, Omidvar et al. 2014, Take 2015, Stanier et al. 2015a). These techniques have been used to observe the macro failure mechanisms of many different geostructures, including for example: pipelines (Dingle et al. 2008); shallow foundations (Mana et al. 2012); jack-up foundations during punch-through (Hu et al. 2014); fault rupture propagation in slopes (Anastasopoulos et al. 2007); slope failure due to seasonal wetting and drying (Take and Bolton, 2011); settlements 
Teng, Y., Stanier, S. A. and Gourvenec, S. M.

'Synchronised multi-scale image analysis of soil deformations'

International Journal of Physical Modelling in Geotechnics

Special edition on DIC. 17(1):53-71 http://dx.doi.org/10.1680/jphmg.15.00058

due to tunneling in sand (Marshall et al. 2012); helical screw piles (Stanier et al. 2013). All of the above examples include PIV/DIC facilitated observations of the macro response of the soil around the geostructures, in some cases with comparison to numerical and/or simple analytical models approximating the mechanisms.

In modern PIV/DIC algorithms (e.g. Pan et al. 2010; Blaber et al. 2015; Stanier et al. 2015a), displacements are generally obtained by:

1) Discretising the region of interest (RoI) within a 'reference' image with a mesh of subsets at a regular spacing interval, then:

2) For each subset:

(i) Calculating a measure of cross-correlation (e.g. normalised cross-correlation; Lewis, 1995) between the 'reference' image and all integer pixel locations within the RoI in the 'target' image.

(ii) Obtaining an initial estimate of the displacement of the subset $(u, v)$ by locating the peak in the cross-correlation map.

(iii) Refining the displacements $(u, v)$ to sub-pixel resolution using a subset shape function to describe the deformation within the subset, achieved by finding optimal values for the subset shape function parameters using an iterative technique (e.g. the InverseCompositional Gauss-Newton (IC-GN) method; Pan et al. 2010) and image intensity interpolation (e.g. bi-quintic b-spline; Cheng et al. 2002). A first-order subset shape function with deformation parameters describing the displacements $(u, v)$ and the displacement gradients $(\partial u / \partial x, \partial v / \partial y, \partial u / \partial y$ and $\partial v / \partial x)$ (Stanier et al. 2015a) is applied.

The above measurement process relies on the assumption that the fraction of FoV within the subset deforms continuously and that the order of that deformation is matched by the order of the subset shape function (Stanier et al. 2015b). In many experimental mechanics applications of PIV/DIC - such as inferring material properties from full-field displacements (Avril et al. 
Teng, Y., Stanier, S. A. and Gourvenec, S. M.

'Synchronised multi-scale image analysis of soil deformations'

International Journal of Physical Modelling in Geotechnics

Special edition on DIC. 17(1):53-71 http://dx.doi.org/10.1680/jphmg.15.00058

2008) - the deformations observed are typically small and strain localisations tend not to occur except perhaps at the very end of a test. However, in geotechnical models the displacements, rotations and deformations are often significantly larger - especially close to the geostructure - making accurate measurement of soil displacements more onerous (see Figure 1). There is also the potential for localisation of the deformations within the body of soil, resulting in high gradients of deformation, at all stages of the experiment.

For granular materials, such as sand, the size of these localisations has been estimated to be of the order of $\sim 10 d_{50}$ (Muir Wood, 2012). It has been established that that images used in PIV/DIC analysis of granular deformation must have image resolution in terms of the particle/pixel size ratio $(d / p)$ of greater than 4 to avoid bias errors and peak locking (Stanier et al. 2015b). This makes the approximate size of a potential localisation in granular materials $\sim 40$ pixels for images with moderate resolution $(d / p \approx 4)$, which is typical of the size of subsets generally used in geotechnical PIV//DIC analyses. In this scenario, many subsets are likely to straddle any localisation features that occur, leading to the subset shape function becoming 'under-matched', that is, unable to approximate the deformation within the subset resulting in rapid decorrelation and bias errors (or drift) in the displacement measurements (Schreier et al. 2002). Much higher resolution images (i.e. $d / p>>4$ ) are likely to be required to measure the deformation occurring within the localisations that occur in granular assemblies.

This paper first describes the hardware and software required to obtain synchronous multi-scale PIV/DIC measurements at moderate and high resolution $(d / p=4$ and 18 respectively). Guidance is then provided on appropriate choices for subset size and spacing with key pitfalls of choosing inappropriate analysis parameters highlighted through examples. A modified photogrammetric correction process to convert the image space (pixel) measurements to model units (mm) - requiring only a small number of static control points - is then proposed and 
Teng, Y., Stanier, S. A. and Gourvenec, S. M.

'Synchronised multi-scale image analysis of soil deformations'

International Journal of Physical Modelling in Geotechnics

Special edition on DIC. 17(1):53-71 http://dx.doi.org/10.1680/jphmg.15.00058

validated. Lastly, experimental data is used to demonstrate the improved resolution of strain field that can be observed using the synchronous multi-scale apparatus.

\section{APPARATUS}

\section{Hardware}

The new synchronised multi-scale image capture system requires minimal additional hardware compared to conventional geotechnical PIV/DIC, requiring only a second camera and control unit that can synchronously trigger both cameras. The multi-scale system hardware is shown in Figure 2. It is a further development on the system described by Stanier and White (2013) and comprises of the following components:

- $\quad$ Centrifuge strongbox - housing the cameras, lighting and soil sample within the PIV strongbox.

- $\quad$ PIV/DIC strongbox - a strongbox that fits within the main centrifuge strongbox that contains the soil sample. A transparent acrylic window is provided to expose a plane of the soil sample allowing digital images to be captured. Arrays of control markers are pre-installed within the window to provide control points with known model space locations for photogrammetric correction of measurements.

- $\quad$ Cameras - two 5-megapixel cameras (Allied Vision Technologies Prosilica GC2450C) with charge coupled device (CCD) sensors and Gigabit Ethernet connectivity. These cameras have a maximum frame rate of 15 frames per second at full resolution. The master camera (viewing the whole, i.e. 'macro' FoV) has an 8-mm lens positioned at the centre of the experimental setup. The slave camera (viewing a subset of the model, the 'micro' FoV) has a 43-mm lens and is positioned adjacent to the master camera. The 8-mm and 43-mm focal lengths were appropriate for the centrifuge PIV strongbox 
Teng, Y., Stanier, S. A. and Gourvenec, S. M.

'Synchronised multi-scale image analysis of soil deformations'

International Journal of Physical Modelling in Geotechnics

Special edition on DIC. 17(1):53-71 http://dx.doi.org/10.1680/jphmg.15.00058

setup used in the beam centrifuge at the University of Western Australia. Different focal lengths may be required for other experimental setups.

- $\quad$ Power supply - an in-house 60V electric power supply is used to support the operation of the cameras and LED panels (not shown in Figure 2 but typically attached to the swing arm of the centrifuge).

- Control units - two control units control the activities of the two cameras. Each unit is connected to a camera and controls the exposure time and frame capture rate. The two control units are connected using an additional cable so that triggering of the two cameras is synchronous, with the 'slave' camera duplicating the activity of the 'master' camera. The controllers also incorporate an LED control unit (Gardasoft RT220) with a LAN interface allowing a TCP/IP link to control the brightness of LED panels independently (see Stanier \& White, 2013).

- $\quad$ Lighting - a pair of light emitting diode (LED) panels (CCS Industries Ltd., Model No. LDL2-266X30SW-WD) are positioned near to the top of the centrifuge strongbox. Light intensity and lighting position can be adjusted to achieve bright and even lighting on the whole FoV using the aforementioned control units.

\section{Software}

The multi-scale PIV/DIC system is controlled by an extension of the software described by Stanier and White (2013). Specifically the software comprises of two packages, written and compiled in the National Instruments LabVIEW environment:

1. DigiCAM - In-house image capturing software. Controls exposure times and frame capture rate whilst providing live video of the camera view. The two cameras are controlled by duplicate interfaces. One instance of the software runs in master mode and controls the 'macro' view camera, whilst a second runs in slave mode, assimilating the various settings directly from the master camera. 
Teng, Y., Stanier, S. A. and Gourvenec, S. M.

'Synchronised multi-scale image analysis of soil deformations'

International Journal of Physical Modelling in Geotechnics

Special edition on DIC. 17(1):53-71 http://dx.doi.org/10.1680/jphmg.15.00058

2. DigiLED - Software to control the light intensity of each of the LED panels to generate even illumination of the model.

Post-processing of the images captured using the two cameras was conducted using the following MATLAB software:

o GeoPIV-RG (available for free download at http://www.geopivrg.com) - PIV/DIC image analysis software incorporating a first-order subset shape function, bi-quintic bspline image intensity interpolation and Inverse Compositional Gauss-Newton (ICGN) subset deformation parameter optimisation. This algorithm provides at least an order of magnitude improvement in measurement precision compared to the PIV/DIC approaches most commonly used in geotechnical applications (Stanier et al. 2015a).

o GeoCALIBRATE_RG_micro - A modified camera calibration script developed to convert pixel measurements derived from images recorded by the slave ('micro' view) camera to model scale units (e.g. mm). Conventional camera calibration techniques (see White et al. 2003) are not applicable for the slave camera due to too few control points being visible in the images recorded, as discussed in detail later in this paper. This newly developed subroutine is available to the geotechnical research community for free download at http://www.geopivrg.com.

\section{EFFECT OF SYSTEM ON IMAGE TEXTURE}

To demonstrate the impact of the higher resolution 'micro' view on the subset texture quality, a physical model test involving vertical penetration of a $50 \mathrm{~mm}$ width $(B), 220 \mathrm{~mm}$ length $(L)$, $10 \mathrm{~mm}$ thick strip foundation was performed on silica sand. The test was performed in the beam centrifuge at UWA under acceleration at the sample surface of 50g. The experimental setup is shown in Figure 2. The 'macro' view camera captures images of a FoV of approximately 350 $\times 300 \mathrm{~mm}$ whilst the 'micro' view camera captures images of a FoV of approximately $80 \times$ 
Teng, Y., Stanier, S. A. and Gourvenec, S. M.

'Synchronised multi-scale image analysis of soil deformations'

International Journal of Physical Modelling in Geotechnics

Special edition on DIC. 17(1):53-71 http://dx.doi.org/10.1680/jphmg.15.00058

60mm. The 5-megapixels ( $2448 \times 2050$ pixels) yield images with pixel density per object unit area of $1045.5 \mathrm{p} / \mathrm{mm}^{2}$ and $47.8 \mathrm{p} / \mathrm{mm}^{2}$ for the 'micro' and 'macro' view images respectively; i.e. a factor of $\sim 20$ difference in resolution.

The soil sample within the strongbox consisted of $\sim 180 \mathrm{~mm}$ depth of UWA medium coarse silica sand ( $d_{50}$ of $510 \mu \mathrm{m}$ ). The combination of the $d_{50}$ of the sand and the FoV of the 'macro' and 'micro' view cameras yield $d_{50} / p$ ratios of $\sim 4$ and $\sim 18$, respectively. This is sufficient to avoid significant 'peak-locking' errors and associated strain field bias errors in the 'macro' view images (Stanier et al. 2015b). For the 'micro' FoV, assuming that potential deformation localisations are of the order of $10 d_{50}$ in size (Muir Wood, 2012), and that a subset 40 pixels in size contains sufficient information for accurate cross-correlation, the analysis subsets will be approximately $\sim 20 \%$ of the size of any localisation features. This potentially allows subsets to populate any localisation features without suffering from excessive decorrelation due to 'undermatching' of the subset shape function.

The sand sample was dry pluviated to a relative density $70 \%$. Prior to pluviation, a fraction of the sand was dyed black and mixed uniformly with undyed sand to enhance the contrast between neighbouring particles. Optimal contrast was achieved by following the procedure recommended by Stanier and White (2013) such that the artificial seeding ratio (ASR) was $~ 0.5$. Images were captured during ramp-up, penetration testing and ramp-down. During the experiment the footing was penetrated into the soil at a rate of $0.1 \mathrm{~mm} / \mathrm{s}$ to a depth of $6.7 \mathrm{~mm}$, hence in this instance the total penetration depth was smaller than the thickness of the foundation. In total 336 images were captured during the footing penetration by each camera at a frequency of $5 \mathrm{~Hz}$.

\section{Subset size and texture quality}

Figure 3 shows the difference in resolution for 'macro' and 'micro' view images for subsets of equivalent model space dimensions (40 pixel diameter in the 'macro' view and 180 pixel 
Teng, Y., Stanier, S. A. and Gourvenec, S. M.

'Synchronised multi-scale image analysis of soil deformations'

International Journal of Physical Modelling in Geotechnics

Special edition on DIC. 17(1):53-71 http://dx.doi.org/10.1680/jphmg.15.00058

diameter in the 'micro' view) noting that circular subsets are utilised in GeoPIV-RG to reduce the potential for measurement bias emanating from subset shape function mismatch. A subset diameter of 40 pixels was chosen for the 'macro' view images, as the subset then contains approximately 100 sand particles, which should provide sufficient information within each subset for accurate cross-correlation (Stanier et al. 2015b). The two subsets, represented by red circles in Figure 3, capture the same surface area of the model in the same location. Individual sand particles can be clearly distinguished in the 'micro' view subset, while similar detail is not discernible in the 'macro' view subset.

To provide a comprehensive analysis of the impact of the 'micro' view on subset texture quality, two subset texture quality parameters have been calculated: (i) the standard deviation of subset pixel intensities ( $\sigma_{I_{s}}$ ) (Stanier and White, 2013); and (ii) the sum of square of subset intensity gradients (SSSIG) (Pan et al. 2008). The former is a simple measure of the contrast within the subset with values of $\sigma_{I_{s}}>15$ indicating adequate contrast (Stanier and White, 2013) whilst the latter is a measure of the distribution of the contrast with values of SSSIG > $10^{5}$ indicating adequate texture distribution (Pan et al. 2008). These measures have been calculated for a range of subsets of varying diameter but the same object space locations (i.e. subset centroid location) for the 'micro' view at two locations. These measures are compared with those for 40 pixel diameter subsets at the same object space locations for the 'macro' view in Figure 4. Red lines mark the adequate subset texture quality thresholds referred to above.

The 40 pixel diameter 'macro' view subsets satisfy both criterions at both locations analysed. For the 'micro' view images, subset diameters greater than 70 pixels also satisfy the criterions at both locations analysed. In general, an appropriately sized subset for image analysis of geotechnical problems should be one that is sufficiently large to contain adequate information for reliable cross-correlation, whilst also being small enough that the shape function adequately captures the deformation within the subset (Stanier et al. 2015b). Therefore, a subset size of 70 
Teng, Y., Stanier, S. A. and Gourvenec, S. M.

'Synchronised multi-scale image analysis of soil deformations'

International Journal of Physical Modelling in Geotechnics

Special edition on DIC. 17(1):53-71 http://dx.doi.org/10.1680/jphmg.15.00058

pixels was deemed optimal for the 'micro' view images as it is the smallest size subset for which there is adequate information within the signal for reliable cross-correlation.

\section{Subset size and correlation quality}

The zero-normalized cross-correlation coefficient $\left(C C_{Z N C C}\right)$ is a robust measure of subset correlation quality that is invariant to global changes in illumination (Pan et al. 2010), with values of 0 and 1 intuitively indicating zero and perfect correlation respectively. It is calculated as:

$$
C C_{Z N C C}=\frac{\sum_{i, j \in n_{s}}\left(I_{i j}^{r}-\bar{I}^{r}\right)\left(I_{i j}^{t}-\bar{I}^{t}\right)}{\sqrt{\sum_{i, j \in n_{s}}\left[I_{i j}^{r}-\bar{I}^{r}\right]^{2} \sum_{i, j \in n_{s}}\left[I_{i j}^{t}-\bar{I}^{t}\right]^{2}}}
$$

where $I_{i j}^{r}$ and $I_{i j}^{t}$ are the pixel intensities for a given $i, j$ coordinate in the 'reference' and 'target' images respectively. The mean pixel intensities for the 'reference' and 'target' images are calculated as:

$$
\bar{I}^{r}=\frac{1}{n_{s}} \sum_{i, j \in n_{s}} I_{i, j}^{r} ; \quad \bar{I}^{t}=\frac{1}{n_{s}} \sum_{i, j \in n_{s}} I_{i, j}^{t}
$$

where $n_{s}$ is the number of pixels in the subset.

The efficacy of PIV/DIC displacement measurement technique relies on the assumption that the deformation within the subset is continuous and of the same order as the shape function used to model the deformation within it, so that the measure of cross correlation defined in Equation 1 can remain close to unity. For granular materials such as sands, any discontinuous deformation (typically caused by particle rearrangement) occurring within a subset results in decorrelation (indicated by in a reduction in $C C_{Z N C C}$ ) due to the subset shape function becoming 'under-matched'. If the decorrelation becomes excessive (i.e. $C C_{Z N C C}$ falls below a user-defined tolerance, $\left.C C_{Z N C C \text {-tol }}\right)$ the 'reference' image must be updated so as to regain adequate crosscorrelation quality. 
Teng, Y., Stanier, S. A. and Gourvenec, S. M.

'Synchronised multi-scale image analysis of soil deformations'

International Journal of Physical Modelling in Geotechnics

Special edition on DIC. 17(1):53-71 http://dx.doi.org/10.1680/jphmg.15.00058

The zero normalised cross-correlation coefficient $\left(C C_{Z N C C}\right)$ was calculated throughout the footing penetration test using GeoPIV-RG for subsets at locations A and B (see Figure 3) of 40 pixel diameter (M40) in the 'macro' view and 180 and 70 pixel diameters ( $\mu 180$ and $\mu 70)$ in the 'micro' view. $C C_{Z N C C-t o l}$ was specified as 0.75 , the maximum number of iterations of the IC-GN optimisation of the deformation parameters for the subset shape function ( max $_{i t e r}$ ) was set to 50 and the maximum difference of the norm of the shape function difference vector $\left(|\Delta p|_{\max }\right)$ was set to $1 \times 10^{-5}$, following the recommendations in Stanier et al. (2015a).

Figure 5 (a \& d) indicates that the decorrelation of the M40 subsets at locations A and B were moderate and thus the 'reference' image did not require updating. However, Figure 5 (b \& e) shows that the $\mu 180$ subset used in the analyses of the 'micro' view images suffered from significant decorrelation. The very sudden decorrelation prior to 'reference' image updating at both locations A and B is indicative of the subset shape function becoming 'under-matched'. That these subsets are of equivalent object space size and utilise the same order subset shape function, illustrates that the 'micro' view images must contain additional information about the deformations occurring within the subsets that is not captured in the lower resolution 'macro' view images.

Figure 4 shows that the $\mu 180$ was potentially larger than necessary as the $\sigma_{I_{s}}$ and SSSIG are both greater than the recommended quality thresholds (Stanier and White, 2013; Pan et al. 2008; Stanier et al. 2015b). Figure 5 (c \& f) illustrates that smaller subsets ( $\mu$ 70) can be used to better analyse the 'micro' images, as the 'reference' image required updating less frequently due to the subset shape function being able to better match the deformations. The correlation coefficients are generally lower for the smaller subset, as for a given $d / p$ ratio, any particle rearrangement has a proportionally greater effect on the correlation coefficient. Nonetheless, despite the enhanced sensitivity to particle rearrangement, there is no evidence of 'undermatched' subset shape function errors. Based on the above observations, the smallest subset 
Teng, Y., Stanier, S. A. and Gourvenec, S. M.

'Synchronised multi-scale image analysis of soil deformations'

International Journal of Physical Modelling in Geotechnics

Special edition on DIC. 17(1):53-71 http://dx.doi.org/10.1680/jphmg.15.00058

with suitable subset quality measures $\left(\sigma_{I_{s}}>15\right.$ and SSSIG $\left.>1 \times 10^{5}\right)$ is recommended for the analysis of high-resolution images, so as to:

1. Minimise the potential for errors due to the subset shape function being 'under-matched' (the effect of under-matching on strain measurements is demonstrated later in this paper); and

2. Ensure that the subset is comfortably smaller than the size of any localisation feature anticipated to develop within the model (i.e. $\left.D_{s}(\mathrm{~mm})<<10 d_{50}\right)$.

\section{The effect of subset size and spacing on strain measurement}

Figure 6 is an illustration of how triplets of subsets (located initially at coordinates $c_{1}, c_{2}$ and $c_{3}$ and finally at $c_{1}{ }^{\prime}, c_{2}{ }^{\prime}$ and $c_{3}{ }^{\prime}$ ), separated by element length $L$, are typically used to calculate measures of strain at the centroid of the triplet (located initially at $E$ and finally at $E^{\prime}$ ) using their displacements $\left(c_{1}-c_{1}{ }^{\prime}, c_{2}-C_{2}\right.$ 'and $\left.c_{3}-C_{3}{ }^{\prime}\right)$. To explore the effect that subset size and spacing has on the strain measurements, triplets of subsets were placed in an equilateral triangle with centroids at locations A and B (see Figure 3) and varying element length $L$. The trajectories of these triplets of subsets were then computed using GeoPIV-RG adopting the same analysis settings as previously, following which the shear $(\gamma)$ and volumetric strains $\left(\varepsilon_{v}\right)$ were calculated following White and Bolton (2004).

Figures 7 and 8 (a \& d) indicate that the element length $L$ has only a minor effect on the measured shear and volumetric strains for the 'macro' view analysis (M40). This demonstrates that the measurements are unaffected by bias due to 'peak locking' (Stanier et al. 2015b). Figures 7 and 8 (b \& e) show that the equivalently sized (in model space terms) 'micro' view analysis $(\mu 180)$ is in general agreement with the 'macro' view analysis with respect to the magnitude of the strains measured, irrespective of the element length $L$. However, there is clear drift in both the shear and volumetric strains at location B, which is caused by the subset shape function becoming 'under-matched' at approximately the same strain magnitudes for all $L$. 
Teng, Y., Stanier, S. A. and Gourvenec, S. M.

'Synchronised multi-scale image analysis of soil deformations'

International Journal of Physical Modelling in Geotechnics

Special edition on DIC. 17(1):53-71 http://dx.doi.org/10.1680/jphmg.15.00058

Figures 7 and 8 (c \& f) show that reducing the subset size to 70 pixels in the 'micro' view $(\mu 70)$ results in very similar shear strain measurements as for the larger subset $(\mu 180)$. However, the volumetric strains - particularly at location A - vary significantly with element length $L$, indicating that the use of smaller subsets and closer spacing (leading to increased measurement density) leads to the measurement of dilation that is masked in the 'macro' view images due to their lower resolution (in terms of $d / p$ ratio), and in the 'micro' view analysis utilising the large subset ( $\mu 180)$ due to the subset shape function becoming 'under-matched'.

Figure 9 (a) shows the shear strain drift for the large subset ( $\mu 180)$ used to compute the shear strain for an element at location B, where $L$ was 160 pixels. The calculated displacement of subset no. 2 drifts erroneously immediately prior to the 'reference' image being updated (Figure $9 \mathrm{c}, \mathrm{d})$, which is a result of the subset shape function becoming 'under-matched' as indicated by corresponding rapid fall in $C C_{Z N C C}$ (Figure $9 \mathrm{~b}$ ). Therefore, it is important to use the smallest subset size exhibiting adequate image texture quality $\left(\sigma_{I_{S}}>15\right.$ and $S S S I G>1 \times 10^{5}$ ) to reduce the potential for the subset shape function becoming 'under-matched' and to carefully explore the effect of subset spacing on the resulting analysis to minimise the potential of bias errors polluting the strain measurements.

\section{PHOTOGRAMMETRIC CORRECTION}

\section{Background}

It is generally preferable for model measurements made using digital images to be converted from image space units (pixels) to more meaningful object space units (e.g. mm). Digital imaging systems typically consist of a small planar sensor (or charge coupled device - CCD) and a magnifying lens assembly. Images captured using such a sensor and magnifying lens assembly are generally polluted by systematic distortions referred to as radial and tangential distortions (Slama, 1980). If a subset moves across a significant proportion of the image width 
Teng, Y., Stanier, S. A. and Gourvenec, S. M.

'Synchronised multi-scale image analysis of soil deformations'

International Journal of Physical Modelling in Geotechnics

Special edition on DIC. 17(1):53-71 http://dx.doi.org/10.1680/jphmg.15.00058

these distortions can lead to significant measurement errors. In addition differential movement of the camera and model during the experiment, such as that observed during centrifuge rampup by White et al. (2003), can lead to further measurement errors. Mathematical models for these distortions and movements have been developed to correct and scale PIV/DIC displacement measurements in a process known as close-range photogrammetry (e.g. Soloff et al. 1997; Heikkilä 2000; White et al. 2003; Pan et al. 2013;).

Close-range photogrammetry generally requires at least 12 static control points - of known image space (typically determined by centroiding) and object space location (measured or prescribed in manufacture) - distributed uniformly across the image, in order to solve the mathematical models for the lens distortions and camera-model movements (rotations and translations) simultaneously by minimising the difference between the modelled and known control point locations. Figure 3 shows that the apparatus described in this paper has adequate control points in the 'macro' FoV; however, only four control points are visible in the 'micro' FoV. So as to minimise the area of the soil sample obscured by static control points, we preferred not to machine additional static control points into the transparent window of the strongbox. Instead an alternate photogrammetric correction process has been developed and validated for the ‘micro' FoV analysis.

\section{Methodology}

The photogrammetric correction method utilised in this paper is based on that described by White et al. (2003). It consists of a pinhole camera model (illustrated in Figure 10) to account for camera-model differential movement (rotations and translations) incorporating functions to correct for four sources of camera-lens induced distortion including: CCD-model nonparallelism, radial distortion, tangential distortion and pixel non-squareness. The equation used to derive object space coordinates $(X, Y)$ from distortion corrected image space coordinates $\left(X_{c}\right.$, $\left.y_{c}\right)$ is as follows: 
Teng, Y., Stanier, S. A. and Gourvenec, S. M.

'Synchronised multi-scale image analysis of soil deformations'

International Journal of Physical Modelling in Geotechnics

Special edition on DIC. 17(1):53-71 http://dx.doi.org/10.1680/jphmg.15.00058

$$
\left[\begin{array}{c}
\mu X \\
\mu Y \\
\mu
\end{array}\right]=P_{R}^{-1} P_{P}^{-1} P_{C}^{-1}\left[\begin{array}{c}
x_{c} \\
y_{c} \\
1
\end{array}\right]
$$

where $\mu$ is a pixel coordinate scale factor and the matrices $P_{R}, P_{C}$ and $P_{P}$ are rotation and translation, camera calibration and perspective project matrices respectively, which are defined as follows:

$$
\begin{gathered}
P_{R}=\left[\begin{array}{lll}
r_{11} & r_{12} & t_{x} \\
r_{21} & r_{22} & t_{y} \\
r_{31} & r_{32} & t_{z}
\end{array}\right] \\
r_{11}=\cos \phi \cos \kappa \\
r_{12}=-\sin \kappa \cos \omega+\cos \kappa \sin \phi \sin \omega \\
r_{21}=\sin \kappa \cos \phi \\
r_{22}=\cos \kappa \cos \omega+\sin \kappa \sin \phi \sin \omega \\
r_{31}=-\sin \phi \\
r_{32}=\cos \phi \sin \omega \\
P_{C}=\left[\begin{array}{ccc}
\frac{1}{D_{x}} & 0 & x_{0} \\
0 & \frac{1}{D_{y}} & y_{0} \\
0 & 0 & 1
\end{array}\right] \\
P_{P}=\left[\begin{array}{ccc}
f & 0 & 0 \\
0 & f & 0 \\
0 & 0 & 1
\end{array}\right] \\
0
\end{gathered}
$$

The distortion corrected image space coordinates $\left(x_{c}, y_{c}\right)$ are derived from the image space coordinates $(x, y)$ as follows: 
Teng, Y., Stanier, S. A. and Gourvenec, S. M.

'Synchronised multi-scale image analysis of soil deformations'

International Journal of Physical Modelling in Geotechnics

Special edition on DIC. 17(1):53-71 http://dx.doi.org/10.1680/jphmg.15.00058

$$
\begin{gathered}
{\left[\begin{array}{c}
x_{c} \\
y_{c}
\end{array}\right]=\left[\begin{array}{c}
\left(\tilde{x}+\delta_{\tilde{x}: r}+\delta_{\tilde{x} t}\right) \frac{1}{D_{x}} \\
\left(\tilde{y}+\delta_{\tilde{y} . r}+\delta_{\tilde{y}: t}\right) \frac{1}{D_{y}}
\end{array}\right]} \\
{\left[\begin{array}{l}
\delta_{\tilde{x} t} \\
\delta_{\tilde{y} y t}
\end{array}\right]=\left[\begin{array}{c}
2 p_{1} \tilde{x} \tilde{y}+p_{2}\left(r^{2}+2 \tilde{x}^{2}\right) \\
p_{1}\left(r^{2}+2 \tilde{y}^{2}\right) 2 p_{2} \tilde{x} \tilde{y}
\end{array}\right]} \\
{\left[\begin{array}{c}
\delta_{\tilde{x}: r} \\
\delta_{\tilde{y}: r}
\end{array}\right]=\left[\begin{array}{c}
\tilde{x}\left(k_{1} r^{2}+k_{2} r^{4}\right) \\
\tilde{y}\left(k_{1} r^{2}+k_{2} r^{4}\right)
\end{array}\right]} \\
{\left[\begin{array}{c}
\tilde{x} \\
\tilde{y}
\end{array}\right]=\left[\begin{array}{l}
\left(x-x_{0}\right) D_{x} \\
\left(y-y_{0}\right) D_{y}
\end{array}\right]} \\
r=\sqrt{\tilde{x} \tilde{x}^{2}+\tilde{y}^{2}}
\end{gathered}
$$

The coordinate scale factor $\mu$ in Equation 3 exists because there is no single solution for the transformation of 2D image space coordinates to 3D object space coordinates; similar but scaled objects appear the same along the optical axis (White, 2002). Therefore, because we only require measurements on a plane (against the transparent window of the strongbox) to be converted from 2D image space coordinates $(x, y)$ to 2D object space coordinates $(X, Y)$ the magnitude of $\mu$ is derived as a product of the matrix multiplication and cancels through division.

Therefore, the photogrammetric system has 14 remaining unknowns that must be optimised for each 'reference' and 'target' image pair. These parameters can be separated into two categories:

1. Camera calibration parameters determined by calibration of the imaging hardware: focal length $(f)$; principal point $\left(x_{0}, y_{0}\right)$; radial lens distortion parameters $\left(k_{1}, k_{2}\right)$; tangential lens distortion parameters $\left(p_{1}, p_{2}\right)$; and CCD non-squareness ratio $(\alpha=$ $D_{y} / D_{x}$, where $D_{x}$ and $D_{y}$ are the dimensions of a pixel on the sensor in object space units). 
Teng, Y., Stanier, S. A. and Gourvenec, S. M.

'Synchronised multi-scale image analysis of soil deformations'

International Journal of Physical Modelling in Geotechnics

Special edition on DIC. 17(1):53-71 http://dx.doi.org/10.1680/jphmg.15.00058

2. Rotation and translation parameters describing the relative position of the camera sensor and model plane: three Euler rotational angles $(\omega, \varphi, \kappa)$; three translation magnitudes $\left(t_{x}, t_{y}, t_{z}\right)$.

Typically, all 14 unknowns are optimised simultaneously by minimising the error between the measured and known coordinates of the static control points. However, this direct one-step approach is not possible for the 'micro' FoV where only four static control points are visible. Instead we propose to pre-calibrate the camera-lens calibration parameters, fixing 8 of the 14 unknowns throughout the experiment. The displacement of the four control points is then used to determine the rotation and translation parameters via optimisation. This alternative process is summarised by the flowchart in Figure 11 . The validity of the approach relies on the assumption that:

1. The nature of the camera-lens induced distortions do not change during the experiment (very unlikely for $1 \mathrm{~g}$ tests but potentially problematic for centrifuge modelling where the lens assembly is subjected to higher stresses); and

2. The rotation and translation parameters can be adequately optimised using displacements of only four static control points.

The following validation will demonstrate that the above assumptions are reasonable even for centrifuge model tests.

\section{Pre-calibration}

The camera calibration parameters $\left(f, x_{0}, y_{0}, k_{1}, k_{2}, p_{1}, p_{2}\right.$ and $\left.\alpha\right)$ were derived using six digital images of a black and white checkerboard that was located in place of the soil sample shown in Figure 2, as illustrated in Figure 12. Each of the images was captured from a slightly different aspect by inclining the checkerboard. The image space coordinates of each of the squares of the checkerboard (48 in total) were extracted using a corner extraction algorithm (Bouguet 2004), as shown for one of the six calibration images in Figure 12 (b). The object space 
Teng, Y., Stanier, S. A. and Gourvenec, S. M.

'Synchronised multi-scale image analysis of soil deformations'

International Journal of Physical Modelling in Geotechnics

Special edition on DIC. 17(1):53-71 http://dx.doi.org/10.1680/jphmg.15.00058

coordinates were specified when printing the checkerboard. These concurrent image and object space coordinates for the six images were then input into a camera calibration algorithm (Heikkilä and Silvén, 1997) to identify the camera calibration parameters and assumed to remain constant throughout the experiment.

\section{Rotation and translation parameter optimisation}

The remaining 6 unknowns in the system of photogrammetric equations (three Euler rotational angles, $\omega, \phi$ and $\kappa$; and three translation magnitudes, $t_{x}, t_{y}$ and $t_{z}$ ) were optimised for each 'reference' and 'target' image pair using the Levenberg-Marquardt least-square fitting algorithm (Moré, 1978) as summarised in the flowchart in Figure 11. The optimisation of the six unknowns was conducted in two-steps:

- Firstly, since the camera was aligned with the model so that the sensor was parallel with the model plane, and the relative rotation of the camera and model was expected to be small, the rotation angles, $\omega, \phi$ and $\kappa$, were assumed to be zero so as to obtain initial estimates of the translation magnitudes, $t_{x}, t_{y}$ and $t_{z}$ that are close to the optimal solution.

- Secondly, all six unknowns are refined using the initial estimates for $t_{x}, t_{y}$ and $t_{z}$ to precondition the system of equations.

This preconditioning process leads to faster and more reliable optimisation of the six imageobject space transformation parameters. In cases where the camera sensor is non-parallel with the model plane, as might be apparent in some experimental setups where the camera cannot be aligned to view the model plane head on, estimates of the initial angles could be used to precondition the system of photogrammetric equations in lieu of the null values we have assumed. 
Teng, Y., Stanier, S. A. and Gourvenec, S. M.

'Synchronised multi-scale image analysis of soil deformations'

International Journal of Physical Modelling in Geotechnics

Special edition on DIC. 17(1):53-71 http://dx.doi.org/10.1680/jphmg.15.00058

\section{Validation}

To validate the above approach, images captured during centrifuge ramp-up by the 'macro' view camera, in which 30 static control points were visible, were used to compare the conventional photogrammetric correction process of White et al. (2003) with the proposed precalibrated approach. Groups of four control points with varying spacing and position were identified in the 'macro' view images, with the control points with the largest spacing in the 'macro' FoV being in broadly the same image space location as the four control points visible in the 'micro' FoV, as illustrated in Figure 13 (a). The measured (via centroiding) image and known object space (measured) coordinates of these groups of four control points were then used in the rotation and translation parameter optimisation process to determine the six remaining unknowns $\left(\omega, \phi, \kappa, t_{x}, t_{y}\right.$ and $\left.t_{z}\right)$, from which the coordinates of the remaining 26 static control points were predicted. The standard deviation of the positional errors (the difference between known and predicted locations) was then calculated throughout the image series for each grouping, as shown in Figure 13 (b) plotted against centrifuge acceleration. These results lead to the following conclusions:

1. The pre-calibrated approach is only slightly less precise than the conventional approach (which has six times the input data available for use in the camera calibration parameter optimisation process).

2. The precision of the pre-calibrated approach is stable throughout the experiment for control point spacing similar to that used in the 'micro' view setup. This indicates that the assumption that the camera-lens distortion parameters can be assumed to remain constant - even during a centrifuge test - is reasonable.

3. The precision of the calibration degrades the closer the four control points are to each other. Therefore, where only a small number of control points are visible, images should be captured such that the control points are in the periphery of the FoV. 
Teng, Y., Stanier, S. A. and Gourvenec, S. M.

'Synchronised multi-scale image analysis of soil deformations'

International Journal of Physical Modelling in Geotechnics

Special edition on DIC. 17(1):53-71 http://dx.doi.org/10.1680/jphmg.15.00058

The modest reduction in precision (in image space terms) between the conventional approach and the proposed pre-calibrated approach is insignificant compared to the gains in measurement resolution that are realised by the use of the 'micro' view camera, as demonstrated in the example below. The four fold reduction in FoV results in object space positional precision in the 'micro' view images that is actually better than that in the 'macro' view, for the control point locations adopted in our apparatus.

\section{EXAMPLE STRAIN FIELD MEASUREMENTS}

The spatial shear and volumetric strain fields generated during the first $0.01 B$ of footing settlement are plotted in Figures 14 and 15 respectively, for both image scales ('macro' and 'micro') and a variety of subset sizes and spacings. For all these analyses the seed correlation

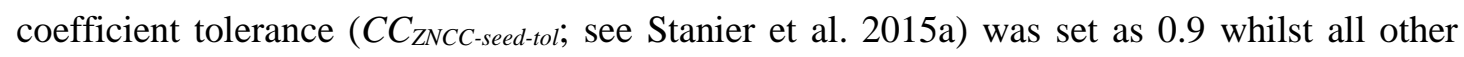
analysis parameters were as defined earlier.

Figures 14 and 15 (a) show the full shear and volumetric strain fields observed by the 'macro' view camera, analysed using a 40 pixel diameter subset $\left(M 40 ; D_{s}=40 p\right)$ with 20 pixel regular gridded spacing $(s=20 p)$. Figures 14 and 15 (b) show a zoomed region of the same 'macro' FoV analysis that is equivalent to the region of the model viewed by the 'micro' FoV. Figure 14 and 15 (c) present the results of an analysis of the 'micro' FoV images using 180 pixel diameter subsets $\left(\mu 180 ; D_{s}=180 p\right)$ at approximately the same object-space spacing as the 'macro' view analysis $(s=80 p)$. Comparing Figures 14 and 15 (b) and (c) shows that the 'macro' and 'micro' FoV analyses with equivalent object space subset size and spacing result in very similar strain fields. However, the 'reference' image had to be updated more regularly for the 'micro' FoV analysis due to periodic rapid decorrelation caused by the subset shape function becoming 'under-matched'. This indicates that the higher resolution $(d / p \approx 18)$ 'micro' view camera is capturing details of deformation processes that are not captured by the 
Teng, Y., Stanier, S. A. and Gourvenec, S. M.

'Synchronised multi-scale image analysis of soil deformations'

International Journal of Physical Modelling in Geotechnics

Special edition on DIC. 17(1):53-71 http://dx.doi.org/10.1680/jphmg.15.00058

comparatively lower resolution $(d / p \approx 4)$ 'macro' view images and that the large subset size precludes their measurement.

Figures 14 and 15 (d) show the effect of reducing the subset size to 70 pixels $\left(\mu 70 ; D_{s}=70 p\right.$ ) in the 'micro' FoV analysis, whilst keeping the subset spacing the same $(s=80 p)$. Immediately beneath the footing a thin band $(\sim 0.06 \mathrm{~B})$ of dilating material is observed in Figure 15 (d) that was not captured by the analyses in Figure 15 (b) and (c). The smaller ( $\mu 70)$ subset is of the order of $\sim 0.04 B$ in size, so the subsets are small enough to populate the dilating feature. In contrast, the larger (M40 and $\mu 180$ ) subsets are of the order of $\sim 0.1 B$ in size, which is significantly larger than the feature observed. This results in subsets straddling the feature, precluding accurate measurement of the deformations occurring within it due to the subset shape becoming 'under-matched'. The smaller subsets are better able to match the deformation within the feature, resulting in less frequent 'reference' image updating and accurate measurement of the deformation.

Lastly, Figures 14 and 15 (e) show the effect of using the same small subset size ( $\mu 70$ ) but with reduced spacing $(s=20 p$ ), which results in a sixteen-fold increase in measurement density. Stanier et al. (2015b) demonstrated that bias errors manifest themselves in strain fields by amplifying strain magnitudes in proportion to the reduction in subset spacing (i.e. strain element gauge length). In this case the same general features and strain magnitudes seen in Figures 14 and 15 (d) are evident, providing confidence that the measurements are not affected by such bias errors. The closer subset spacing results in strain element sizes much smaller than the strain features being observed in the experiment, yielding comparatively more detailed strain fields than the analysis with larger subset spacing (c.f. subfigures (d) and (e) in Figures 14 and 15). This analysis confirms that for this experimental setup and granular material, the optimum subset size is $\sim 70$ pixels and subset spacing is $\sim 20$ pixels. 
Teng, Y., Stanier, S. A. and Gourvenec, S. M.

'Synchronised multi-scale image analysis of soil deformations'

International Journal of Physical Modelling in Geotechnics

Special edition on DIC. 17(1):53-71 http://dx.doi.org/10.1680/jphmg.15.00058

For different experimental setups and soils it is recommended to carefully determine appropriate subset sizes by: (i) assessing subset texture quality for a wide range of subset sizes and (ii) exploring the effect of subset spacing (i.e. strain element gauge length) on the strain measurements that are derived from the displacement fields. The use of higher resolution images $(d / p>>4)$, the smallest subset size exhibiting adequate image texture quality and close spacing results in the ability to observe small localised strain features that are either not captured by low resolution images $(d / p \approx 4)$ or are masked by the subset shape function becoming 'under-matched' in analyses performed on high resolution images $(d / p \approx 18)$ using subsets that are larger than necessary.

\section{CONCLUSIONS}

A multi-scale image analysis system has been described that can capture images of both the 'macro' failure mechanism and 'micro' scale deformation localisations using a pair of synchronously triggered digital cameras viewing different regions of a geotechnical model test. The hardware and software required for such a system has been described. Guidance has been given on appropriate analysis parameter choice, including subset size and spacing. Pitfalls caused by inappropriate choice of those user-defined analysis parameters have been described and demonstrated using example data from a centrifuge test modelling the settlement of a strip footing on silica sand. A modified photogrammetric correction procedure to convert image space (pixel) measurements to model units (e.g. mm) - requiring as few as four static control points to be visible within the images being analysed - has been proposed. This procedure has been validated using images obtained during centrifuge ramp-up, illustrating that it is reasonable to assume that camera calibration parameters can remain constant throughout an experiment with minimal loss of positional accuracy and precision. Lastly, the effect of image scale, subset size and spacing on strain field measurements has been demonstrated. High resolution $(d / p \approx 18)$ images analysed using the smallest subset exhibiting adequate image 
Teng, Y., Stanier, S. A. and Gourvenec, S. M.

'Synchronised multi-scale image analysis of soil deformations'

International Journal of Physical Modelling in Geotechnics

Special edition on DIC. 17(1):53-71 http://dx.doi.org/10.1680/jphmg.15.00058

texture quality measures and close spacing, results in highly detailed strain fields that are either not captured by low resolution images $(d / p \approx 4)$ or are masked in analyses of high resolution images that utilise subsets that are larger than necessary.

\section{ACKNOWLEDGEMENTS}

This work forms part of the activities of the Centre for Offshore Foundation Systems (COFS). Established in 1997 under the Australian Research Council’s Special Research Centres Program. Supported as a node of the Australian Research Council's Centre of Excellence for Geotechnical Science and Engineering, and through the Fugro Chair in Geotechnics, the Lloyd's Register Foundation Chair and Centre of Excellence in Offshore Foundations and the Shell EMI Chair in Offshore Engineering. The third author and the work presented in this paper are supported through the Australian Research Council’s Centre of Excellence for Geotechnical Science and Engineering (CE110001009). This support is gratefully acknowledged. Special thanks also to Mr Kelvin Leong (Senior Centrifuge Technician) for his assistance during the experiments; to Mr Shane De Catania (Senior Electronics Technician) for the software developments; and to Professor David White for his advice on developing the 'micro' FoV camera calibration procedure.

\section{NOMENCLATURE}

$\begin{array}{ll}\alpha & \text { Pixel non-squareness ratio } \\ \text { ASR } & \text { artificial seeding ratio } \\ B & \text { footing width } \\ C & \text { initial coordinates of subset } \\ C^{\prime} & \text { final coordinates of subset } \\ C C_{Z N C C} & \text { zero normalised cross-correlation correlation coefficient } \\ C_{\text {ZNCC-min-tol }} & \text { full field correlation coefficient tolerance }\end{array}$


Teng, Y., Stanier, S. A. and Gourvenec, S. M.

'Synchronised multi-scale image analysis of soil deformations'

International Journal of Physical Modelling in Geotechnics

Special edition on DIC. 17(1):53-71 http://dx.doi.org/10.1680/jphmg.15.00058

CC $C_{\text {ZNCC-seed-tol }}$ seed correlation coefficient tolerance

$\delta_{\tilde{x}: r} \quad$ horizontal error due to radial lens distortion

$\delta_{\tilde{x}: t} \quad$ horizontal error due to tangential lens distortion

$\delta_{\bar{y} r \boldsymbol{r}} \quad$ vertical error due to radial lens distortion

$\delta_{\bar{y}: t} \quad$ vertical error due to tangential lens distortion

diameter

$d_{50} \quad$ median particle diameter

$D_{s} \quad$ diameter of GeoPIV-RG subset

$D_{x} \quad$ width of a pixel on the camera sensor in object space units

$D_{y} \quad$ height of a pixel on the camera sensor in object space units

E initial strain element centroid

$E^{\prime} \quad$ final strain element centroid

f camera focal length

$k_{1} \quad$ radial lens distortion parameter

$k_{2} \quad$ radial lens distortion parameter

$\kappa \quad$ Euler rotation angle between the $N$ axis and the $X$ axis

L gauge length

M40 40 pixel diameter subset in 'macro’ view

$\mu \quad$ coordinate scale factor

$\mu 70 \quad 70$ pixel diameter subset in 'micro' view

$\mu 180$ pixel diameter subset in 'micro' view

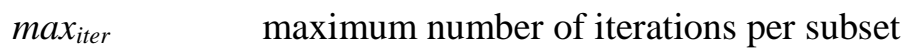

NCC normalised cross correlation

$\omega \quad$ Euler rotation angle between the $X^{\prime}$ axis and the $N$ axis

$|\Delta p|_{\max } \quad$ maximum norm of the shape function difference vector 
Teng, Y., Stanier, S. A. and Gourvenec, S. M.

'Synchronised multi-scale image analysis of soil deformations'

International Journal of Physical Modelling in Geotechnics

Special edition on DIC. 17(1):53-71 http://dx.doi.org/10.1680/jphmg.15.00058

pixel

$p_{1}$

tangential lens distortion parameter

$p_{2}$

tangential lens distortion parameter

$\varphi$

Euler rotation angle between the $Z$ ' axis and the $Z$ axis

$r$

radius

$\sigma_{I_{s}}$

standard deviation of subset pixel intensities

S

subset spacing

SSSIG

sum of the square of subset intensity gradients

$t_{X}$

translation magnitude

$t_{y}$

translation magnitude

$t_{z}$

translation magnitude

$u$

horizontal displacement in image space

v

vertical displacement in image space

X

horizontal position in image space

$x_{0}$

horizontal position of principal point of camera in image space

$\tilde{\boldsymbol{x}}$

horizontal position in camera sensor space w. central origin

X

horizontal position in object space units

y

vertical position in image space

$y_{0}$

vertical position of principal point of camera in image space

$\tilde{\boldsymbol{y}}$

vertical position in camera sensor space w. central origin

Y

vertical position in object space units 
Teng, Y., Stanier, S. A. and Gourvenec, S. M.

'Synchronised multi-scale image analysis of soil deformations'

International Journal of Physical Modelling in Geotechnics

Special edition on DIC. 17(1):53-71 http://dx.doi.org/10.1680/jphmg.15.00058

\section{REFERENCES}

Adrian, R. J. (1991). Particle-imaging techniques for experimental fluid mechanics. Annual review of fluid mechanics, 23(1): 261-304.

Adrian, R. J., and Westerweel, J. (2011). Particle image velocimetry: Cambridge University Press.

Anastasopoulos, I., Gazetas, M., Bransby, M., Davies, M., \& El Nahas, A. (2007). Fault rupture propagation through sand: finite-element analysis and validation through centrifuge experiments. Journal of Geotechnical and Geoenvironmental Engineeering 133: 943-958.

Avril, S., Bonnet, M., Brettelle, A-S.,Grédiac, M., Hild, F., Ienny, P., Latourte, F., Lemosse, D., Pagano, S., Pagnacco, E., Pierron, F. (2008). Overview of identification methods of mechanical parameters based on full-field measurements. Experimental Mechanics, 48: 381-402.

Bouguet, J.-Y. (2004). Camera calibration toolbox for matlab. Available for download at http://www.vision.caltech.edu/bouguetj/calib_doc.

Cheng, P., Sutton, M.A., Schreier, H.W. and McNeill, S.R. (2002). Full field speckle pattern image correlation with b-spline deformation function. Experimental Mechanics, 42(3): 344-352.

Dingle, H.R.C., White, D.J., Gaudin, C. (2008). Mechanisms of pipe embedment and lateral breakout on soft clay. Canadian Geotechnical Journal, 45(5): 636-652.

Hall, S. (2012). Digital Image Correlation in Experimental Geomechanics. ALERT Geomaterials Doctoral Summer School 2012. ALERT Geomaterials. ISBN: 978-3-00039683-0. pp. 69-102.

Heikkilä, J. (2000). Geometric camera calibration using circular control points. IEEE Transactions on Pattern Analysis and Machine Intelligence. 22(10): 1066-1077. 
Teng, Y., Stanier, S. A. and Gourvenec, S. M.

'Synchronised multi-scale image analysis of soil deformations'

International Journal of Physical Modelling in Geotechnics

Special edition on DIC. 17(1):53-71 http://dx.doi.org/10.1680/jphmg.15.00058

Heikkilä, J., and Silvén, O. (1997). A four-step camera calibration procedure with implicit image correction. Computer Vision and Pattern Recognition, 1997. Proceedings of the 1997 IEEE Computer Society Conference on (pp. 1106-1112): IEEE.

Hu, P., Wang, D., Cassidy, M.J. and Stanier, S.A. (2014). Assessing the punch-through hazard of a spudcan on sand overlying clay. Géotechnique, 65(11): 1-14.

Iskander M. (2010) Modelling in transparent soils. Springer. ISBN-13: 978-3642025006.

Mana, D. S. K., Gourvenec, S., and Martin, C. M. (2012). Critical skirt spacing for shallow foundations under general loading. Journal of geotechnical and geoenvironmental engineering, 139(9), 1554-1566.

Marshall, A., Farrel, R., Klar, A., and Mair, R. (2012). Tunnels in sands: the effect of size, depth and volume loss on greenfield displacements. Géotechnique. 62(5): 385-399.

Moré, J. J. (1978). The Levenberg-Marquardt algorithm: implementation and theory Numerical analysis (pp. 105-116): Springer.

Muir Wood, D. M., (2012). Heterogeneity and Soil Element Testing. Géotechnique Letters, 2: $101-106$.

Omidvar, M., Chen, Z. and Iskander, M. (2014). Image-base Lagrangian analysis of granular kinematics. Journal of Computing in Civil Engineering, 29(6): 1-16.

Pan, B., Xie, H., and Wang, Z. (2010). Equivalence of digital image correlation criteria for pattern matching. Applied optics, 49(28), 5501-5509.

Pan, B., Xie, H., Wang, Z., Qian, K., and Wang, Z. (2008). Study on subset size selection in digital image correlation for speckle patterns. Optics express, 16(10), 7037-7048.

Pan, B., Yu, L., Wu, D., and Tang, L. (2013). Systematic errors in two-dimensional digital image correlation due to lens distortion. Optics and lasers in engineering, 51(2), 140-147.

Rechenmacher, A.L. and R.J. Finno (2004). Digital image correlation to evaluate shear banding in dilative sands. ASTM Geotechnical Testing Journal, 27 (1), 13-22. 
Teng, Y., Stanier, S. A. and Gourvenec, S. M.

'Synchronised multi-scale image analysis of soil deformations'

International Journal of Physical Modelling in Geotechnics

Special edition on DIC. 17(1):53-71 http://dx.doi.org/10.1680/jphmg.15.00058

Schreier, H.W. and Sutton, M.A. (2002). Systematic errors in digital image correlation due to under matched subset shape functions. Experimental Mechanics, 42(3): 303-310.

Soloff, S. M., Adrian, R. J., and Liu, Z.-C. (1997). Distortion compensation for generalized stereoscopic particle image velocimetry. Measurement science and technology, 8(12), 1441.

Slama, C. (1980). Manual of photogrammetry (4th Edition), American Society of Photogrammetry: 105 North Virginia Avenue, Falls Church, VA.

Stanier, S.A., Blaber, J., Take, W., and White, D. (2015a). Improved image-based deformation measurement for geotechnical applications. Canadian Geotechnical Journal, doi: 10.1139/cgj-2015-0253.

Stanier, S.A., Dijkstra, J., Leśniewska, D., Hambleton, J., White, D.J. and Muir Wood, D. (2015b). Vermiculate artefacts in image analysis of granular materials. Computers and Geotechnics, doi:10.1016/j.compgeo.2015.11.013.

Stanier, S.A., Black, J.A. and Hird, C.C. (2013). Modelling helical screw piles in soft clay and design implications. ICE Geotechnical Engineering, 167(5):447-460.

Stanier, S. A., and White, D. J. (2013). Improved image-based deformation measurement in the centrifuge environment. Geotechnical Testing Journal, 36(6), 1-14.

Sutton, M. A., McNeill, S. R., Helm, J. D., and Chao, Y. J. (2000). Advances in twodimensional and three-dimensional computer vision Photomechanics (pp. 323-372): Springer.

Sutton, M. A., Wolters, W. J., Peters, W. H., Ranson, W. F., and McNeill, S. R. (1983). Determination of displacements using an improved digital correlation method. Image and vision computing, 1(3), 133-139.

Take, W.A. (2015). Thirty-Sixth Canadian Geotechnical Colloquium: Advances in Visualization of Geotechnical Processes through Digital Image Correlation. Canadian Geotechnical Journal, doi: 10.1139/cgj-2014-0080. 
Teng, Y., Stanier, S. A. and Gourvenec, S. M.

'Synchronised multi-scale image analysis of soil deformations'

International Journal of Physical Modelling in Geotechnics

Special edition on DIC. 17(1):53-71 http://dx.doi.org/10.1680/jphmg.15.00058

Take, W. and Bolton, M. (2011). Seasonal rathceting and softening in clay slopes, leading to first-time failure. Géotechnique, 61(9): 757-769.

Teh, K. L., Cassidy, M. J., Leung, C. F. et al. (2008). Revealing the bearing capacity mechanisms of a penetrating spudcan through sand overlying clay. Géotechnique 58(10): 793-804.

Westerweel, J. (1997). Fundamentals of digital particle image velocimetry. Measurement science and technology, 8(12), 1379.

White, D. (2002). An investigation into the behaviour of pressed-in piles, $\mathrm{PhD}$ thesis, University of Cambridge.

White, D. J., and Bolton, M. D. (2004). Displacement and strain paths during plane-strain model pile installation in sand. Geotechnique, 54(6), 375-397.

White, D. J., Take, W. A., and Bolton, M. D. (2003). Soil deformation measurement using particle image velocimetry (PIV) and photogrammetry. Geotechnique, 53(7), 619-631.

White D. J., Take W.A, Bolton M.D. \& Munachen S.E. (2001) A deformation measuring system for geotechnical testing based on digital imaging, close-range photogrammetry, and PIV image analysis. Proc. 15th Int. Conf. on Soil Mechanics and Geotechnical Engineering. Istanbul, Turkey. pp 539-542. Balkema, Rotterdam. 
Teng, Y., Stanier, S. A. and Gourvenec, S. M.

'Synchronised multi-scale image analysis of soil deformations'

International Journal of Physical Modelling in Geotechnics

Special edition on DIC. 17(1):53-71 http://dx.doi.org/10.1680/jphmg.15.00058

\section{FIGURES}

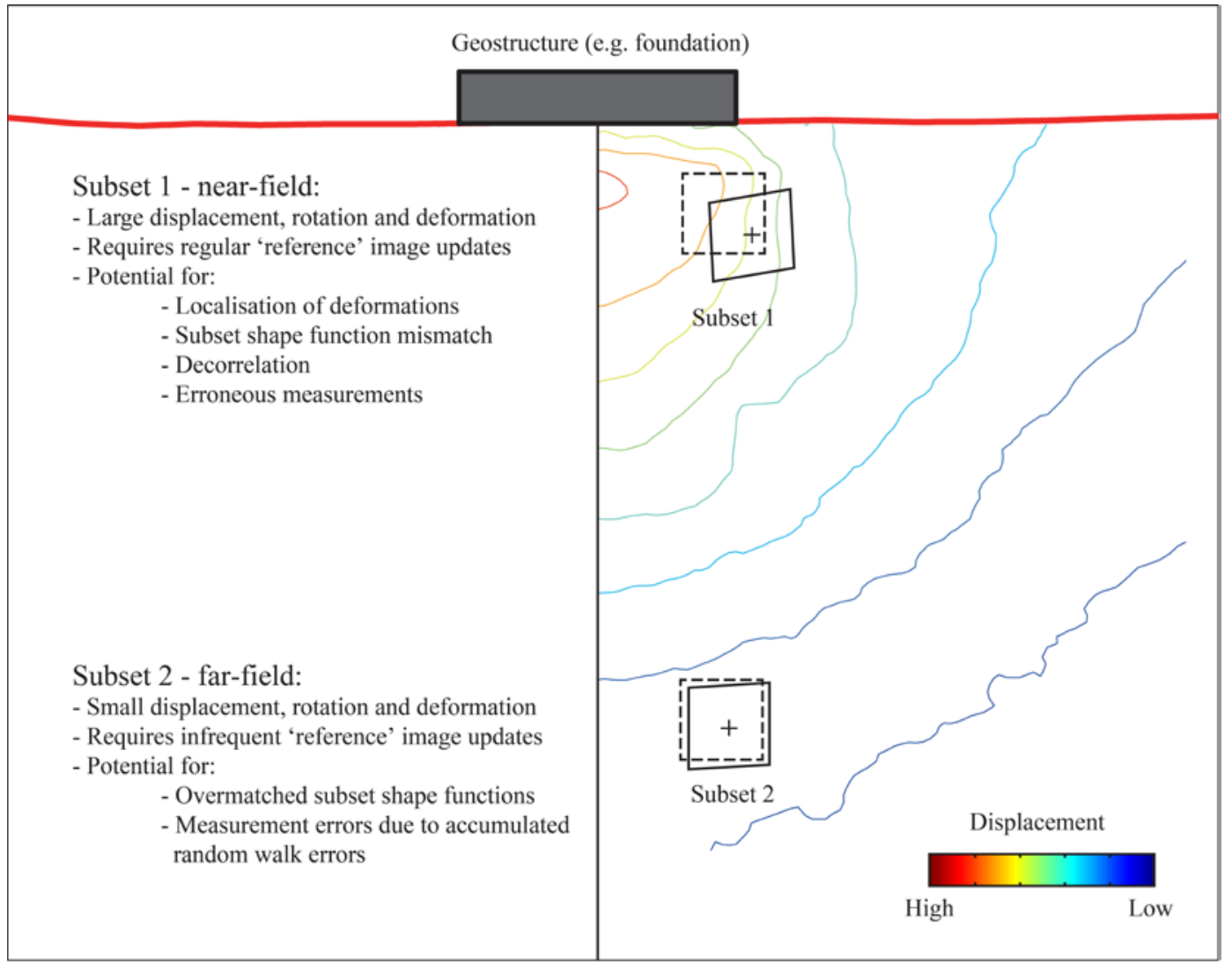

Figure 1: Schematic of a typical geotechnical model test incorporating PIV/DIC measurements

illustrating the range of subset displacements, rotations and deformation experienced across the field of view of the digital camera. 
Teng, Y., Stanier, S. A. and Gourvenec, S. M.

'Synchronised multi-scale image analysis of soil deformations'

International Journal of Physical Modelling in Geotechnics

Special edition on DIC. 17(1):53-71 http://dx.doi.org/10.1680/jphmg.15.00058

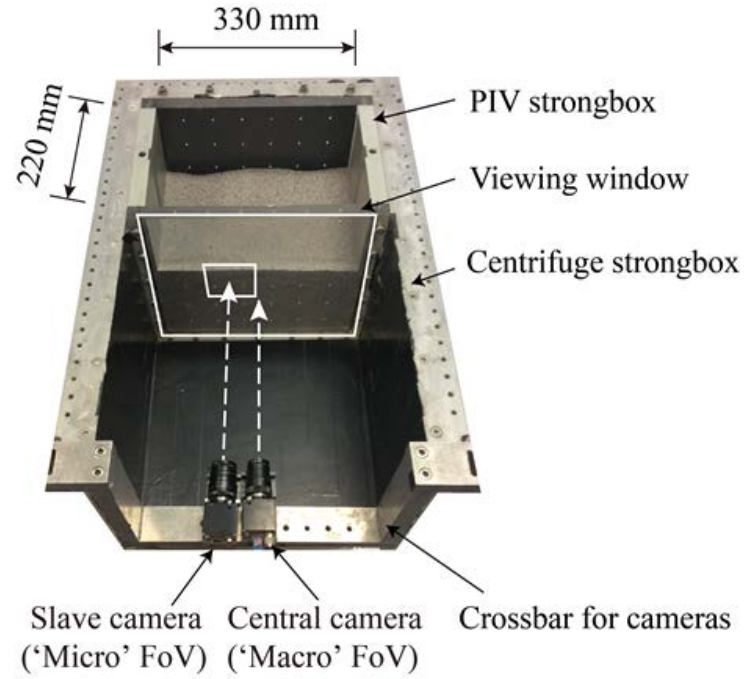

Figure 2: Synchronous multi-scale PIV/DIC apparatus developed for the UWA beam centrifuge.

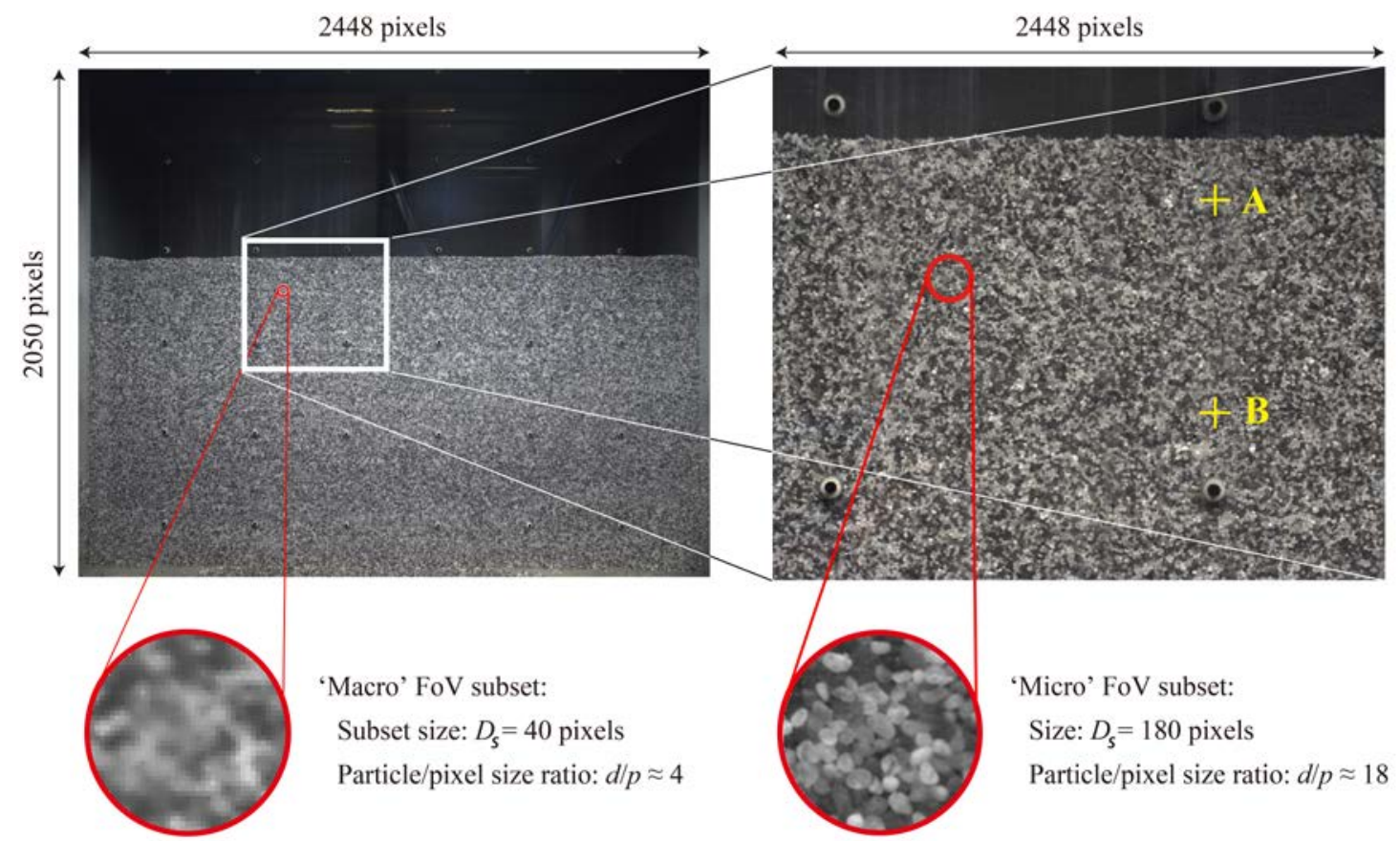

Figure 3: Example images captured using the ‘macro' (LHS) and ‘micro' (RHS) FoVs with magnified view of example subsets to illustrate the difference in image resolution. 
Teng, Y., Stanier, S. A. and Gourvenec, S. M.

'Synchronised multi-scale image analysis of soil deformations'

International Journal of Physical Modelling in Geotechnics

Special edition on DIC. 17(1):53-71 http://dx.doi.org/10.1680/jphmg.15.00058

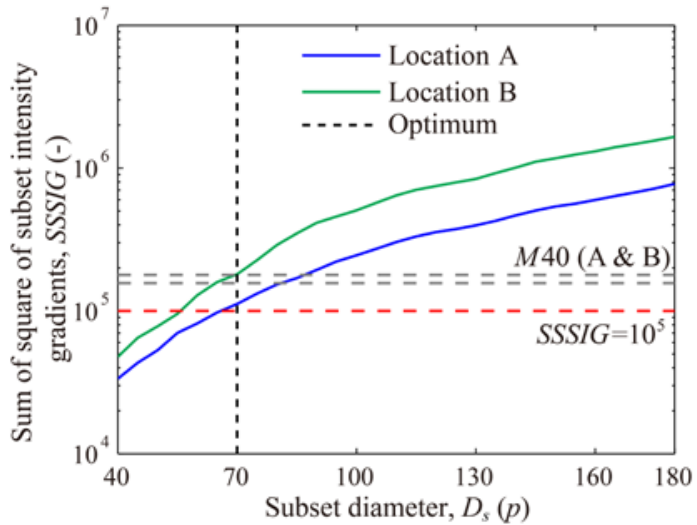

(a)

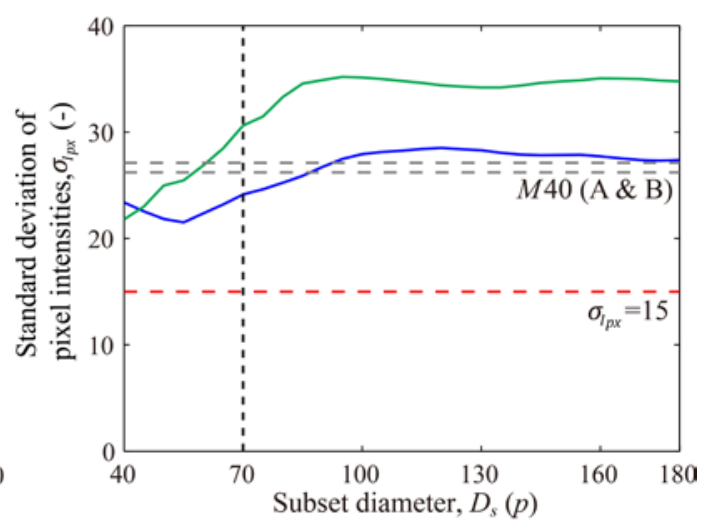

(b)

Figure 4: Measures of subset quality for a range of subset diameters for the 'micro' FoV compared to 40 pixel diameter subset for the 'macro' view: (a) standard deviation of pixel intensities $\left(\sigma_{I_{p x}}\right)$; and (b) Sum of Square of Subset Intensity Gradient (SSSIG).

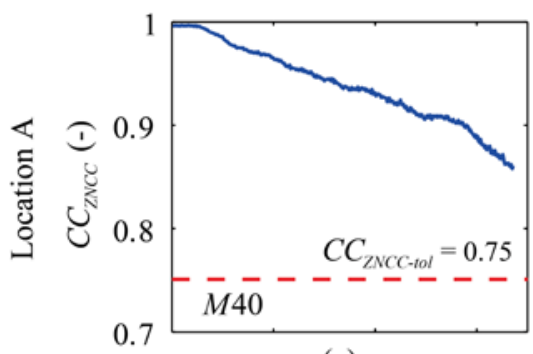

(a)

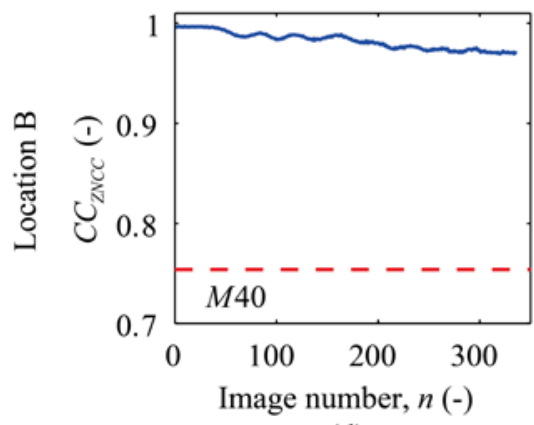

(d)

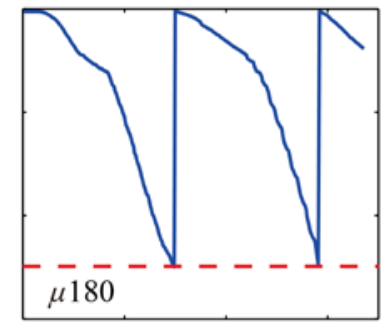

(b)

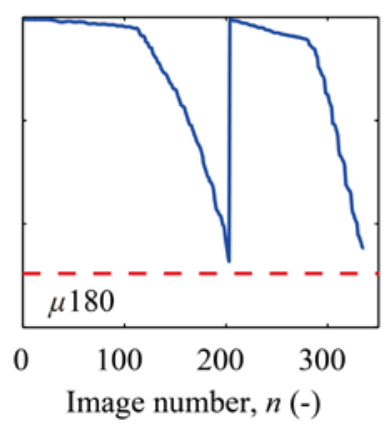

(e)

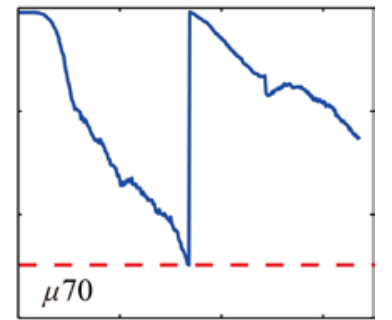

(c)

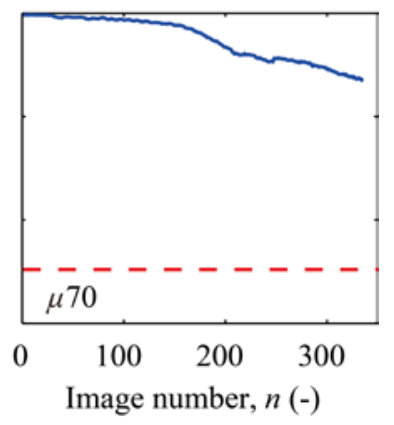

(f)

Figure 5: Decorrelation for subset locations A and B (see Figure) for: (a,d) 40 pixel 'macro' view subset (M40); (b,e) 180 pixel 'micro' view subset ( $\mu 180)$; and (c,f) 70 pixel 'micro' view subset $(\mu 70)$. 
Teng, Y., Stanier, S. A. and Gourvenec, S. M.

'Synchronised multi-scale image analysis of soil deformations'

International Journal of Physical Modelling in Geotechnics

Special edition on DIC. 17(1):53-71 http://dx.doi.org/10.1680/jphmg.15.00058

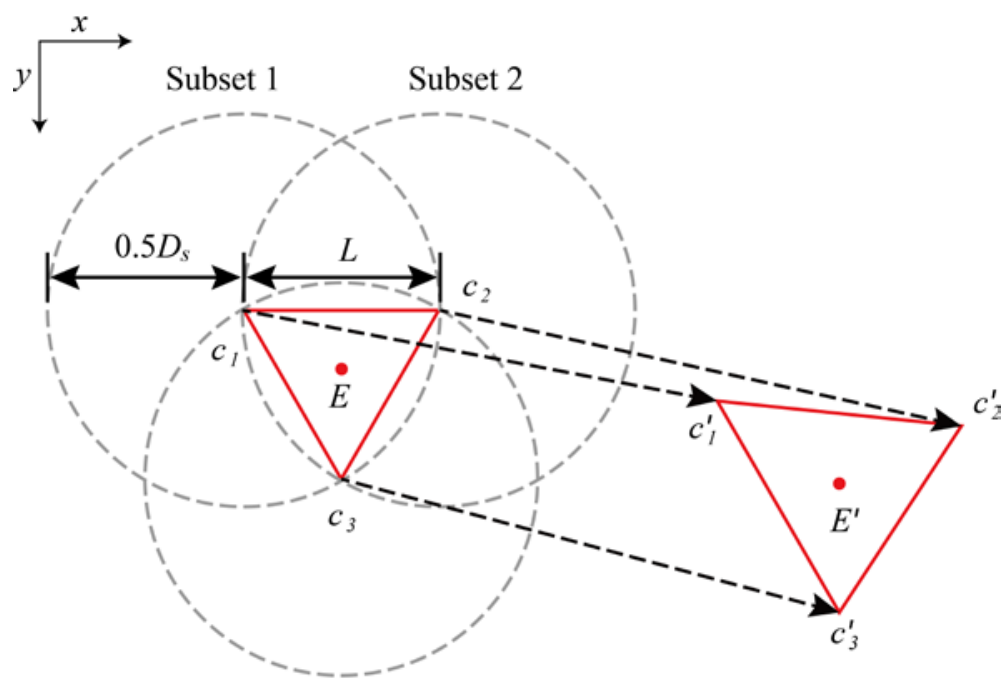

Subset 3

Figure 6: Schematic of strain element geometry with centroid located at E and E’ comprising of a triangle of subsets with centroids located at $c_{1}, c_{2}, c_{3}, c_{1}{ }^{\prime}, c_{2}{ }^{\prime}$ and $c_{3}{ }^{\prime}$. 
Teng, Y., Stanier, S. A. and Gourvenec, S. M.

'Synchronised multi-scale image analysis of soil deformations'

International Journal of Physical Modelling in Geotechnics

Special edition on DIC. 17(1):53-71 http://dx.doi.org/10.1680/jphmg.15.00058

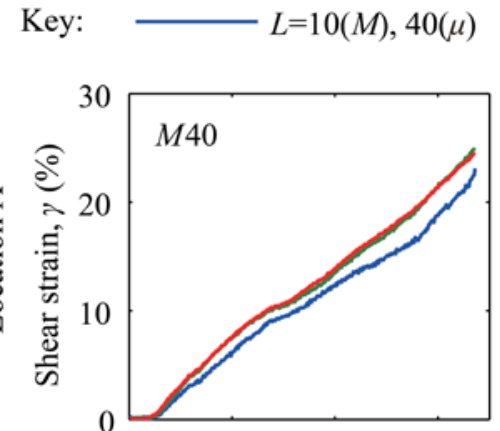

(a)

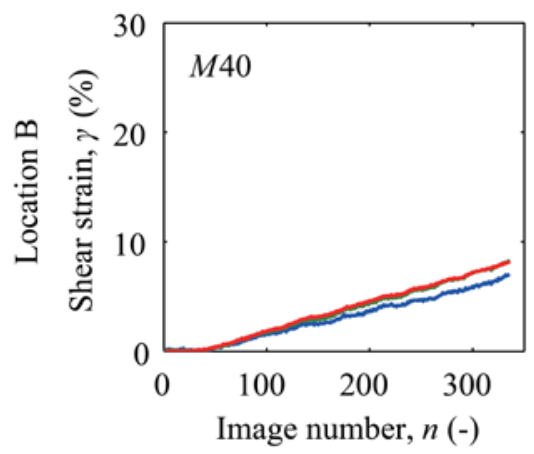

(d)

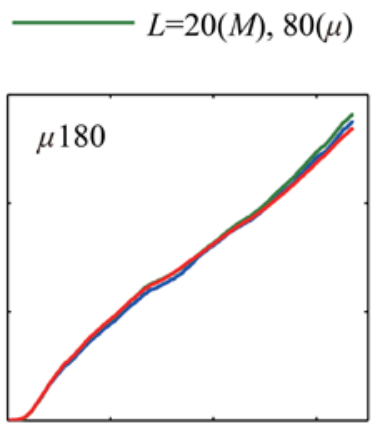

(b)

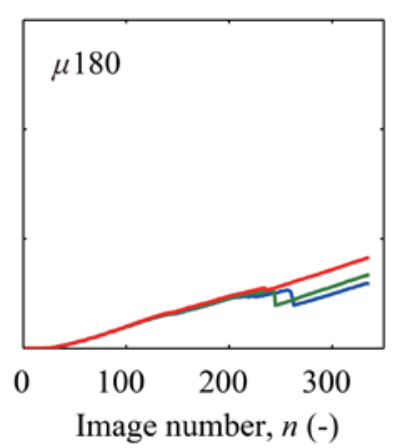

(e)

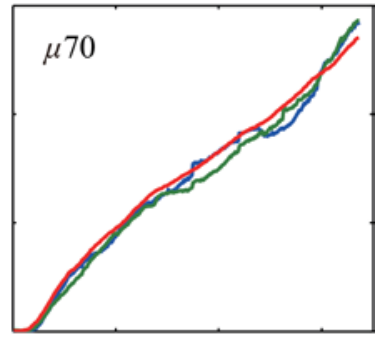

(c)

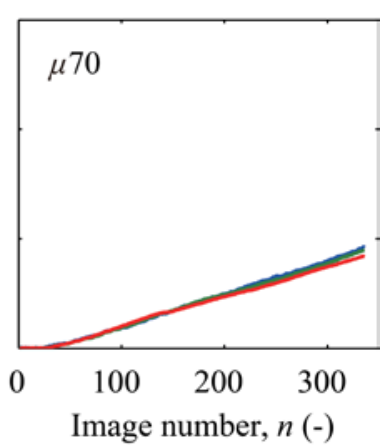

(f)

Figure 7: Shear strain $(\gamma)$ measured using triangular elements of varying element length $L$ at locations A and B for: (a,d) 40 pixel diameter subset in 'macro' view (M40); (b,e) 180 pixel diameter subset in 'micro' view ( $\mu 180)$; and 70 pixel diameter subset in ‘micro' view $(\mu 70)$. 
Teng, Y., Stanier, S. A. and Gourvenec, S. M.

'Synchronised multi-scale image analysis of soil deformations'

International Journal of Physical Modelling in Geotechnics

Special edition on DIC. 17(1):53-71 http://dx.doi.org/10.1680/jphmg.15.00058

Key: $\longrightarrow L=10(M), 40(\mu)$

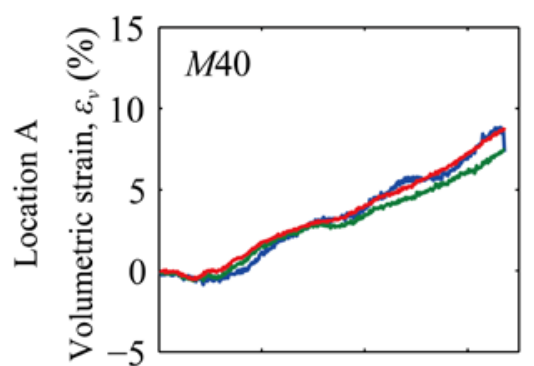

(a)

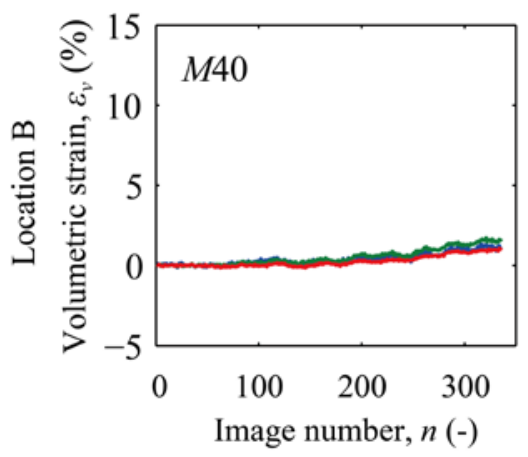

(d)

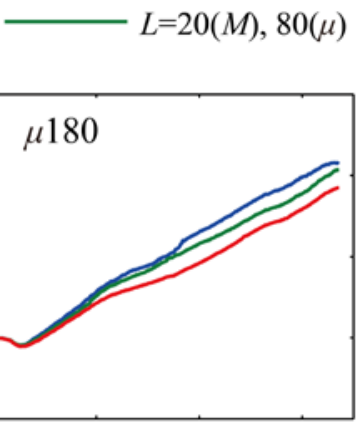

(b)

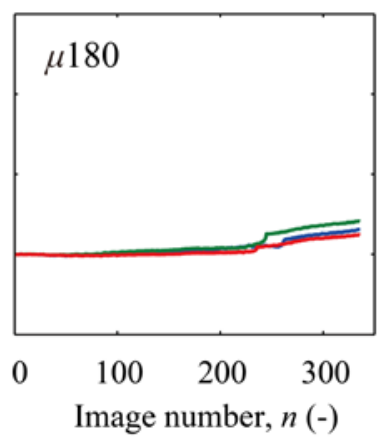

(e)

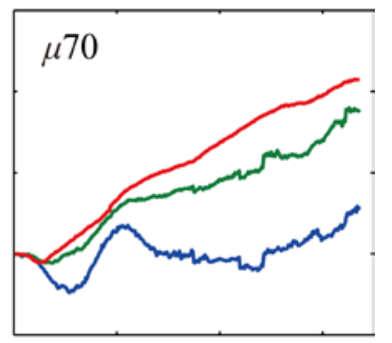

(c)

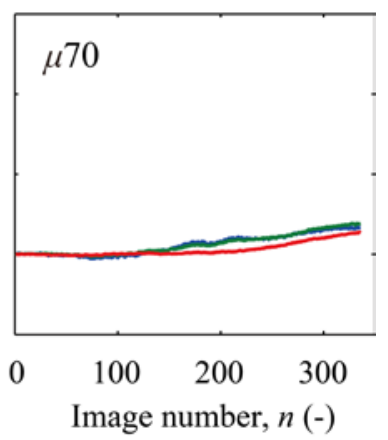

(f)

Figure 8: Volumetric strain $\left(\varepsilon_{v}\right)$ measured using triangular elements of varying element length $L$ at locations A and B for: (a,d) 40 pixel diameter subset in 'macro' view (M40); (b,e) 180 pixel diameter subset in 'micro' view ( $\mu 180)$; and 70 pixel diameter subset in 'micro' view $(\mu 70)$. 
Teng, Y., Stanier, S. A. and Gourvenec, S. M.

'Synchronised multi-scale image analysis of soil deformations'

International Journal of Physical Modelling in Geotechnics

Special edition on DIC. 17(1):53-71 http://dx.doi.org/10.1680/jphmg.15.00058

Key: $\mathbf{\nabla}$ Start of measurement drift

- End of measurement drift

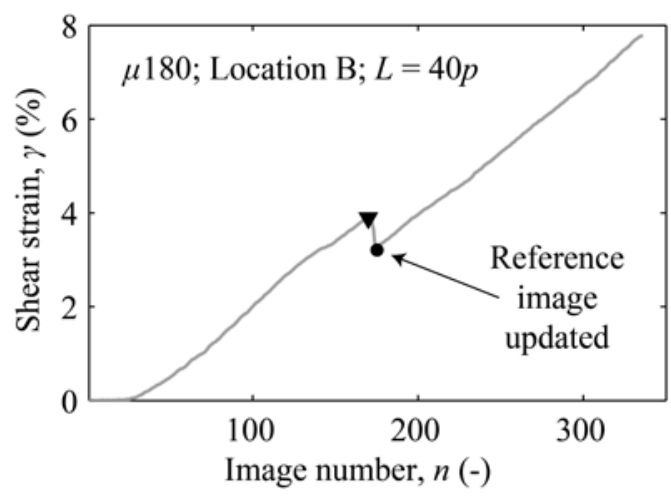

(a)

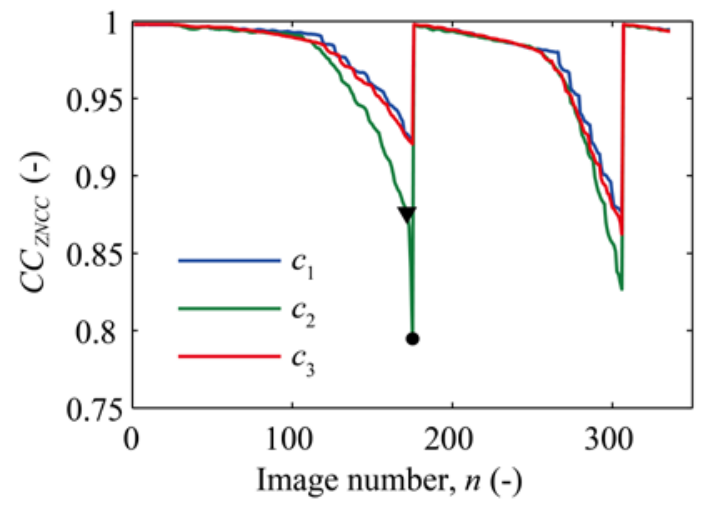

(b)

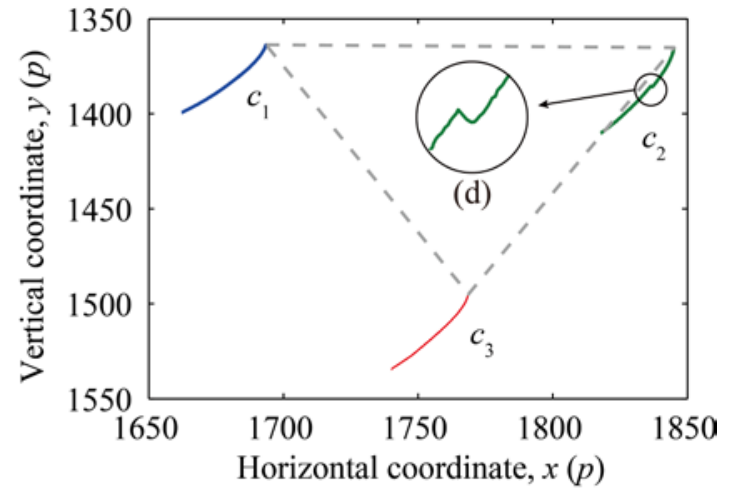

(c)

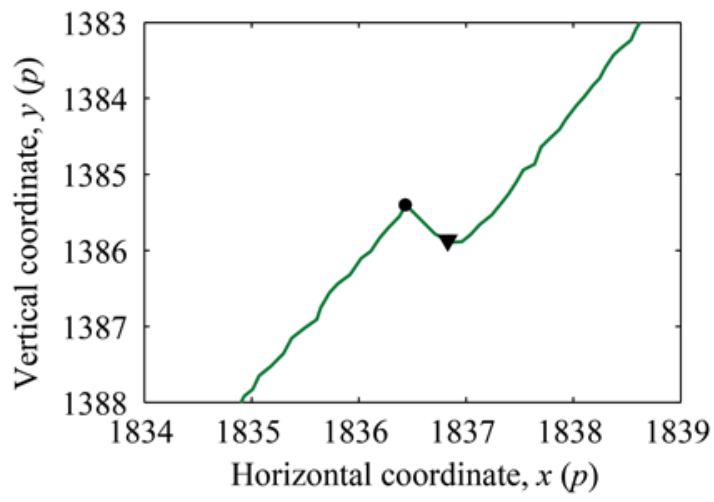

(d)

Figure 9: Effect of subset shape function mismatch on strain computations: (a) sudden shear strain drift caused by (b) sudden decorrelation due to subset shape function mismatch immediately prior to 'reference' image updating, resulting in (c,d) erroneous displacement measurement drift, which pollutes the strain computations. 
Teng, Y., Stanier, S. A. and Gourvenec, S. M.

'Synchronised multi-scale image analysis of soil deformations'

International Journal of Physical Modelling in Geotechnics

Special edition on DIC. 17(1):53-71 http://dx.doi.org/10.1680/jphmg.15.00058

Object space frame $(\mathrm{mm})$

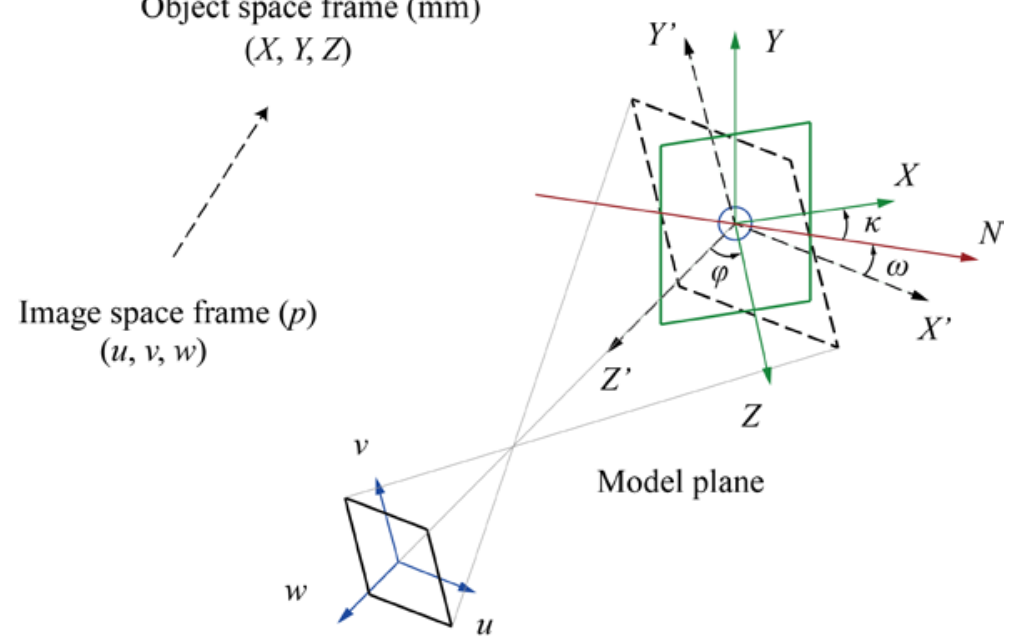

Camera sensor

Transformation of $u, v$ to $X, Y$ performed using Equations 3-11:

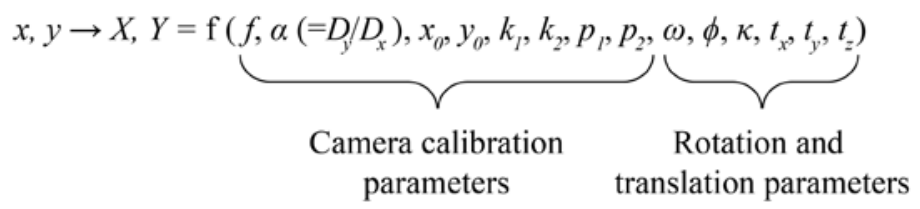

Figure 10: Pinhole camera model for converting image space measurements $(x, y)$ to object space measurements $(X, Y)$. 
Teng, Y., Stanier, S. A. and Gourvenec, S. M.

'Synchronised multi-scale image analysis of soil deformations'

International Journal of Physical Modelling in Geotechnics

Special edition on DIC. 17(1):53-71 http://dx.doi.org/10.1680/jphmg.15.00058

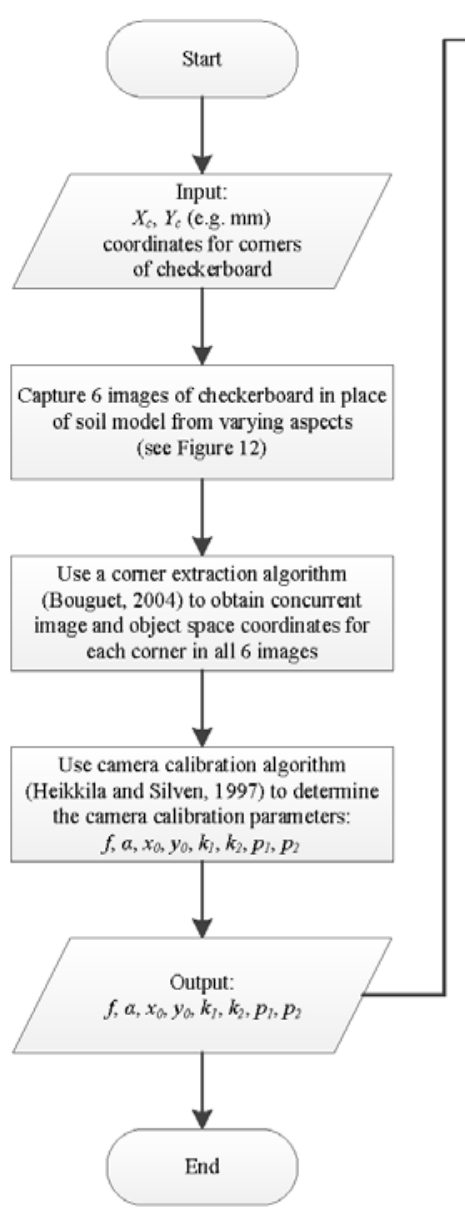

(a)

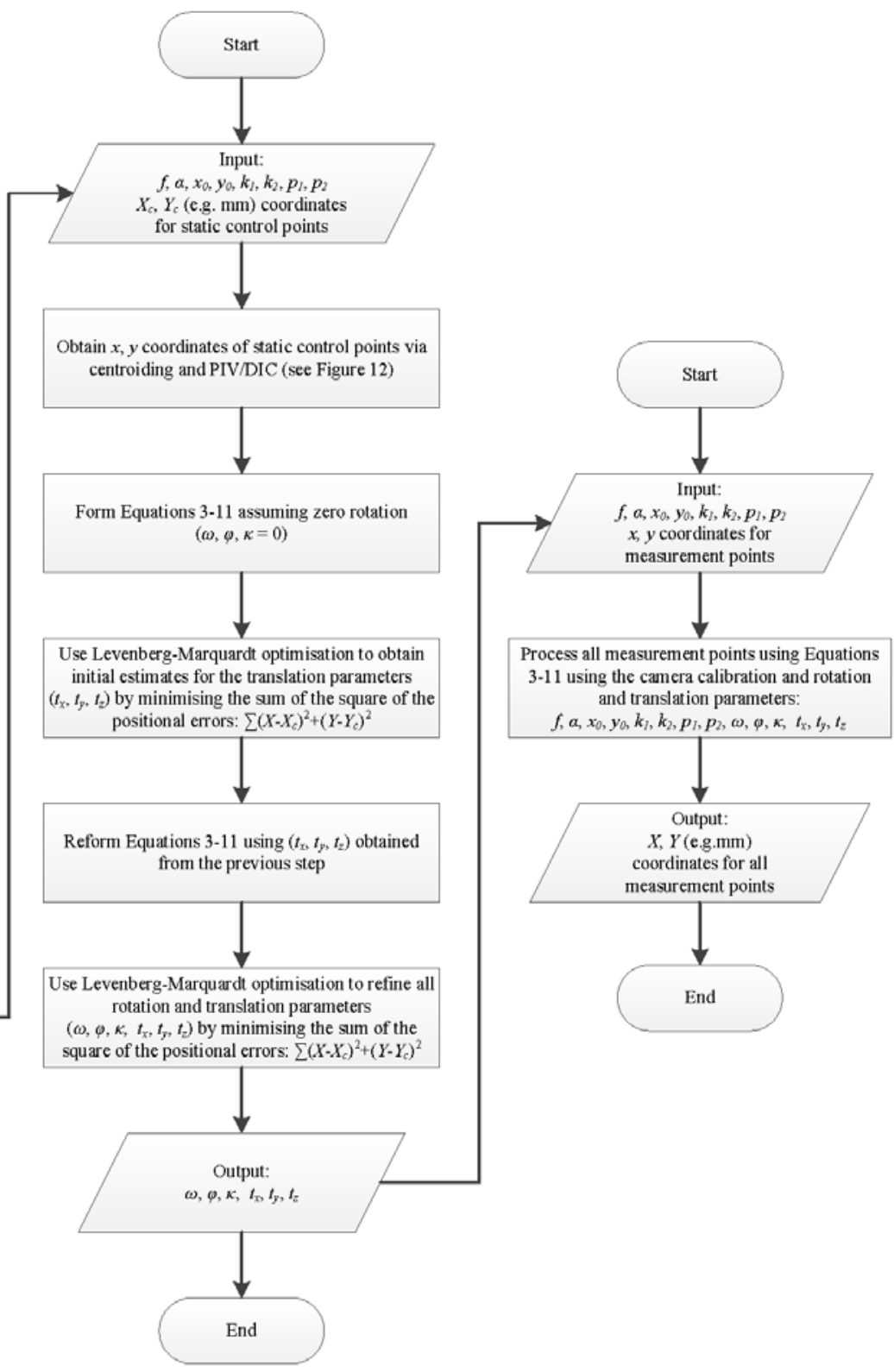

(b) (c)

Figure 11: Flowchart detailing the steps involved in the proposed pre-calibrated

photogrammetric correction process: (a) camera calibration; (b) rotation and translation parameter optimisation; and (c) measurement point processing. 
Teng, Y., Stanier, S. A. and Gourvenec, S. M.

'Synchronised multi-scale image analysis of soil deformations'

International Journal of Physical Modelling in Geotechnics

Special edition on DIC. 17(1):53-71 http://dx.doi.org/10.1680/jphmg.15.00058

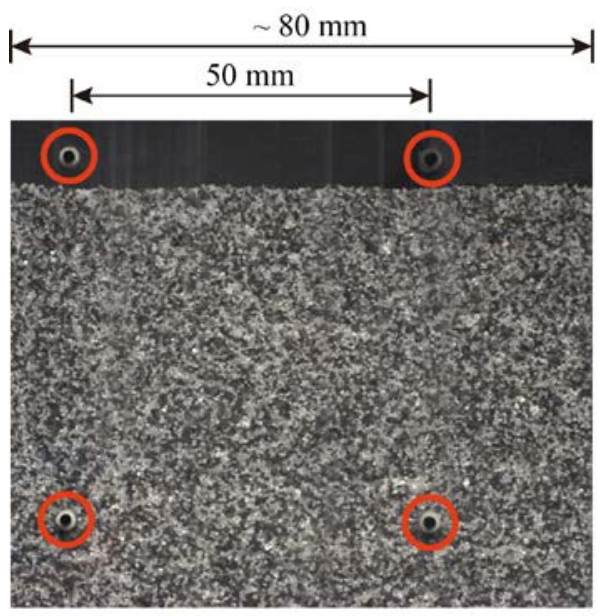

(a)

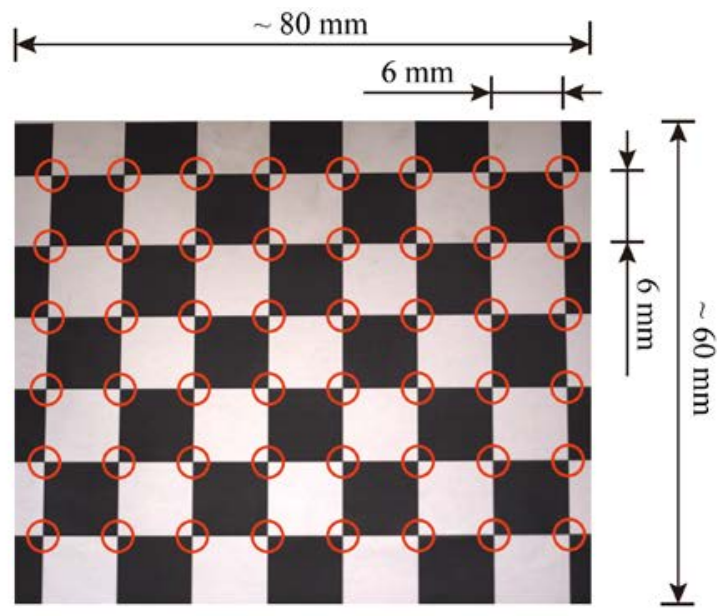

(b)

Figure 12: Pre-calibration of the camera calibration parameters using a checkerboard in place of the soil sample and corner extraction: (a) ‘micro' view of soil sample with four static control points circled; and (b) checkerboard used in place of soil sample during calibration with corners circled. 
Teng, Y., Stanier, S. A. and Gourvenec, S. M.

'Synchronised multi-scale image analysis of soil deformations'

International Journal of Physical Modelling in Geotechnics

Special edition on DIC. 17(1):53-71 http://dx.doi.org/10.1680/jphmg.15.00058

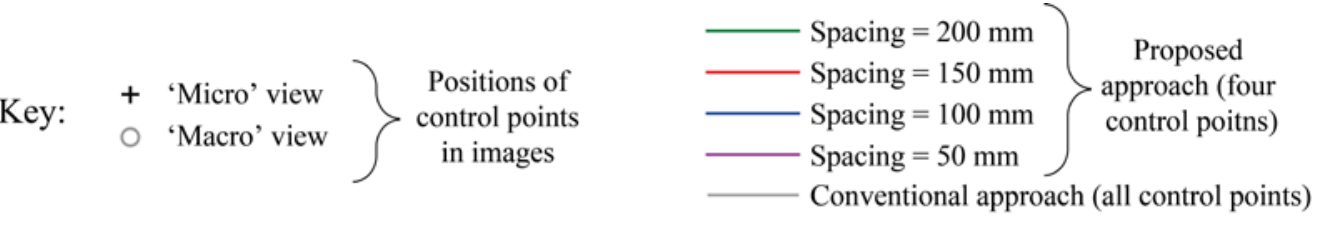

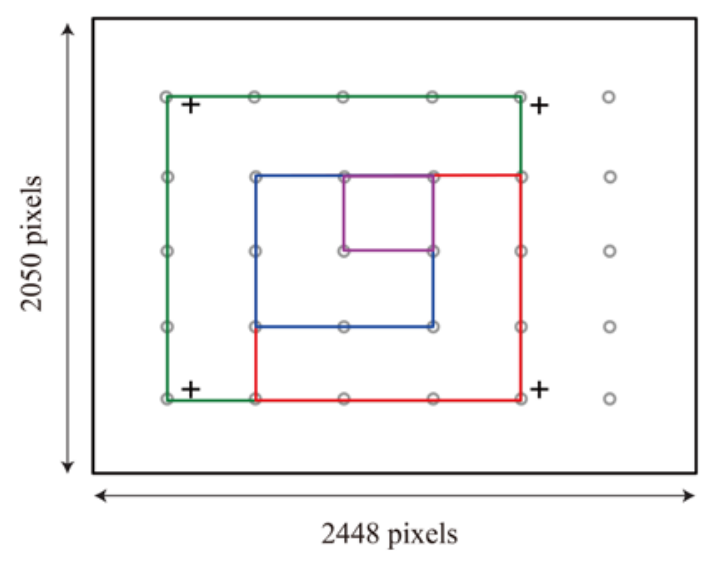

(a)

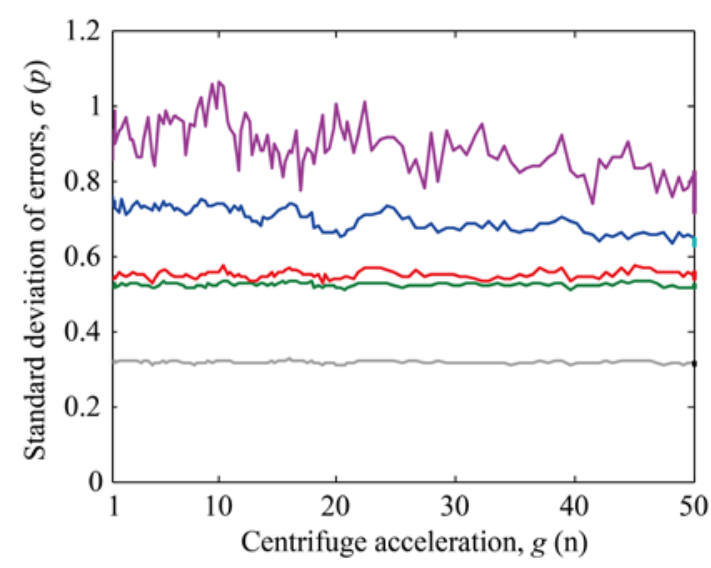

(b)

Figure 13: Pre-calibrated photogrammetric correction validation: (a) location of sets of four control points in the 'macro' view images used in the validation process compared to the locations of those available in the analysis of the 'micro' view images; and (b) precision of the proposed pre-calibrated photogrammetric correction process compared to the conventional approach during centrifuge ramp-up. 
Teng, Y., Stanier, S. A. and Gourvenec, S. M.

'Synchronised multi-scale image analysis of soil deformations'

International Journal of Physical Modelling in Geotechnics

Special edition on DIC. 17(1):53-71 http://dx.doi.org/10.1680/jphmg.15.00058
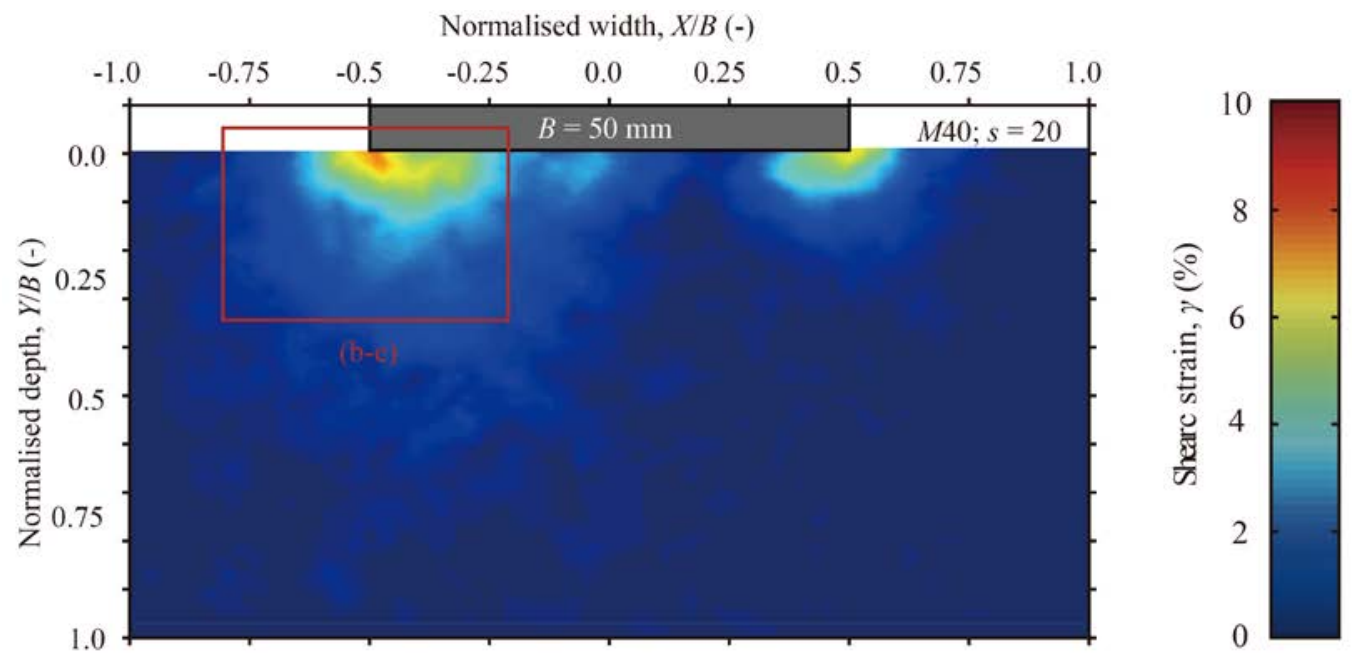

(a)

Normalised width, $X / B(-)$

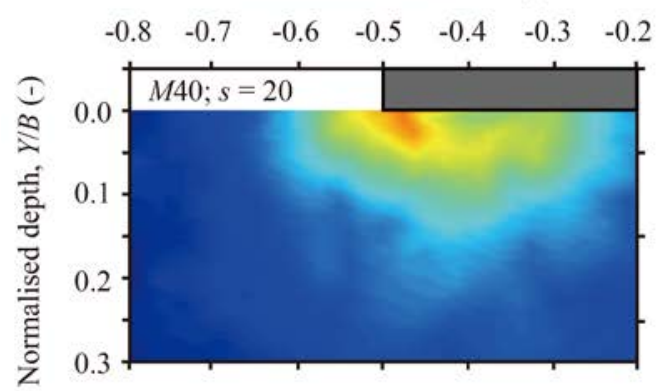

(b)

Normalised width, $X / B(-)$

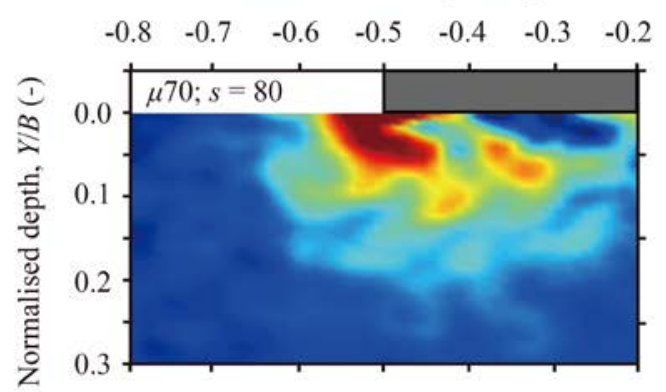

(d)

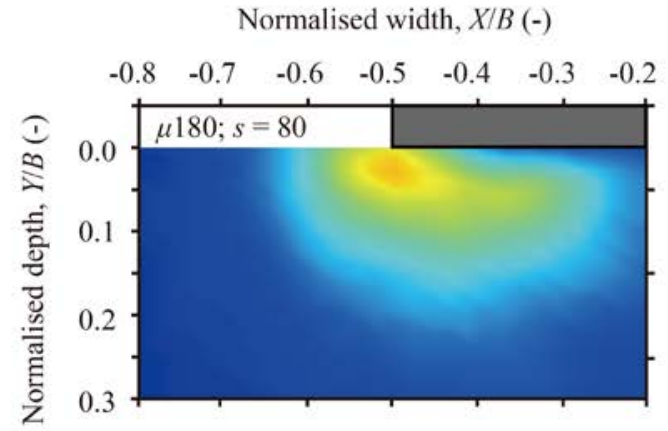

(c)

Normalised width, $X / B(-)$

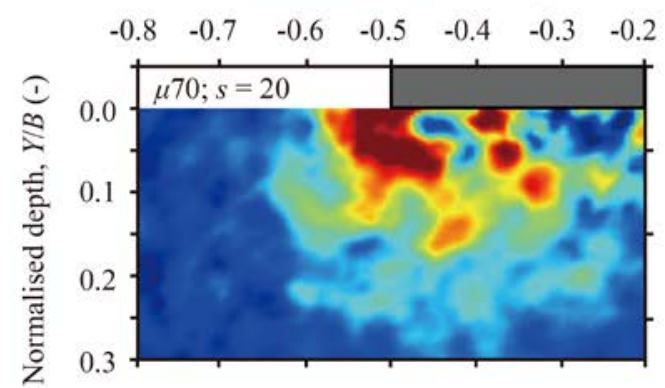

(e)

Figure 14: Shear strain fields $(\gamma)$ beneath a strip footing penetrating into dense silica sand: (a) 'macro' view for $D_{s}=40 p$; $s=20 p$; (b) zoomed region of 'macro' view equivalent to 'micro' view; (c) 'micro’ view for $D_{s}=180 p$; $s=80 p$; (d) 'micro' view for $D_{s}=70 p$; $s=80 p$; and (e) ‘micro' view for $D_{s}=70 p ; s=20 p$. 
Teng, Y., Stanier, S. A. and Gourvenec, S. M.

'Synchronised multi-scale image analysis of soil deformations'

International Journal of Physical Modelling in Geotechnics

Special edition on DIC. 17(1):53-71 http://dx.doi.org/10.1680/jphmg.15.00058

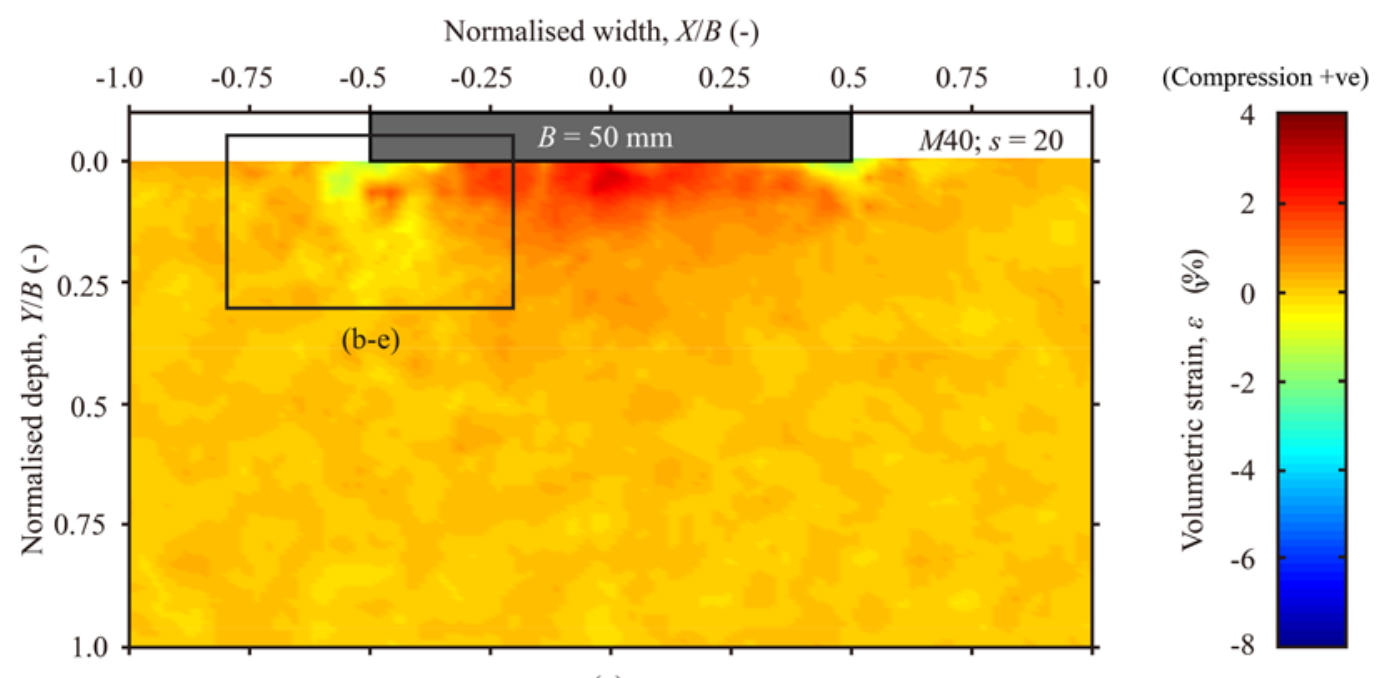

(a)

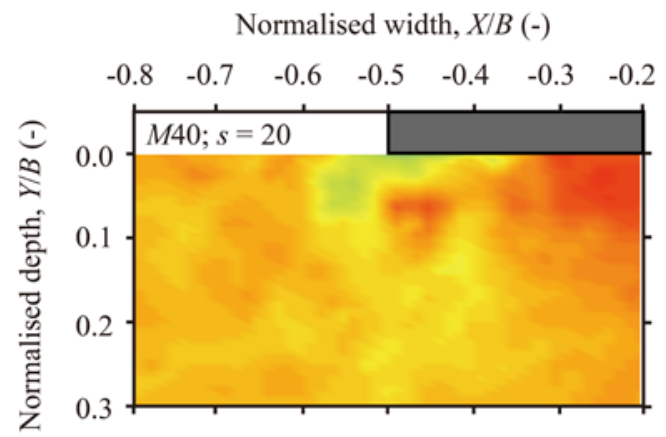

(b)

Normalised width, $X / B(-)$

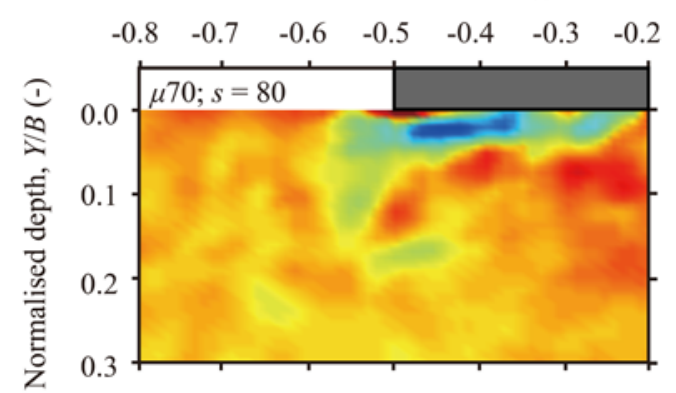

(d)

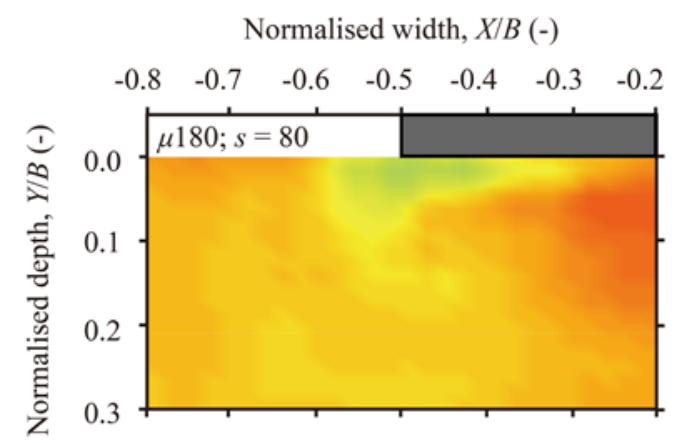

(c)

Normalised width, $X / B(-)$

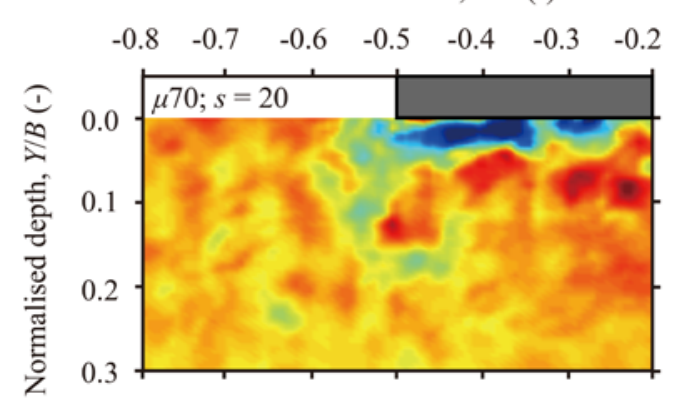

(e)

Figure 15: Volumetric strain fields $\left(\varepsilon_{v}\right)$ beneath a strip footing penetrating into dense silica sand: (a) 'macro' view for $D_{s}=40 p$; $s=20 p$; (b) zoomed region of 'macro' view equivalent to ‘micro' view; (c) ‘micro' view for $D_{s}=180 p$; $s=80 p$; (d) 'micro' view for $D_{s}=70 p$; $s=$ 80p; and (e) ‘micro' view for $D_{s}=70 p$; $s=20 p$. 